\title{
Prototype Open Event Reconstruction Pipeline for the Cherenkov Telescope Array
}

\author{
M. Nöthe ${ }^{a, *}$, K. Kosack ${ }^{b}$, L. Nickel ${ }^{a}$ and M. Peresano ${ }^{b}$ for the CTA Consortium \\ ${ }^{a}$ TU Dortmund University, \\ Otto-Hahn-Str. 4a, Dortmund, Germany \\ ${ }^{b}$ AIM, CEA, CNRS, Universite Paris-Saclay, Universite Paris Diderot, Sorbonne Paris Cite, F-91191 \\ Gif-sur-Yvette, France \\ E-mail: maximilian.noethe@tu-dortmund.de
}

The Cherenkov Telescope Array (CTA) is the next-generation gamma-ray observatory currently under construction. It will improve over the current generation of imaging atmospheric Cherenkov telescopes (IACTs) by a factor of five to ten in sensitivity and it will be able to observe the whole sky from a combination of two sites: a northern site in La Palma, Spain, and a southern one in Paranal, Chile. CTA will also be the first open gamma-ray observatory. Accordingly, the data analysis pipeline is developed as open-source software. The event reconstruction pipeline accepts raw data of the telescopes and processes it to produce suitable input for the higher-level science tools. Its primary tasks include reconstructing the physical properties of each recorded shower and providing the corresponding instrument response functions.

ctapipe is a framework providing algorithms and tools to facilitate raw data calibration, image extraction, image parameterization and event reconstruction. Its main focus is currently the analysis of simulated data but it has also been successfully applied for the analysis of data obtained with the first CTA prototype telescopes, such as the Large-Sized Telescope 1 (LST-1).

pyirf is a library to calculate IACT instrument response functions, needed to obtain physics results like spectra and light curves, from the reconstructed event lists.

Building on these two, protopipe is a prototype for the event reconstruction pipeline for CTA. Recent developments in these software packages will be presented.

$37^{\text {th }}$ International Cosmic Ray Conference (ICRC 2021)

July $12^{\text {th }}-23^{\text {rd }}, 2021$

Online - Berlin, Germany

\footnotetext{
* Presenter
} 


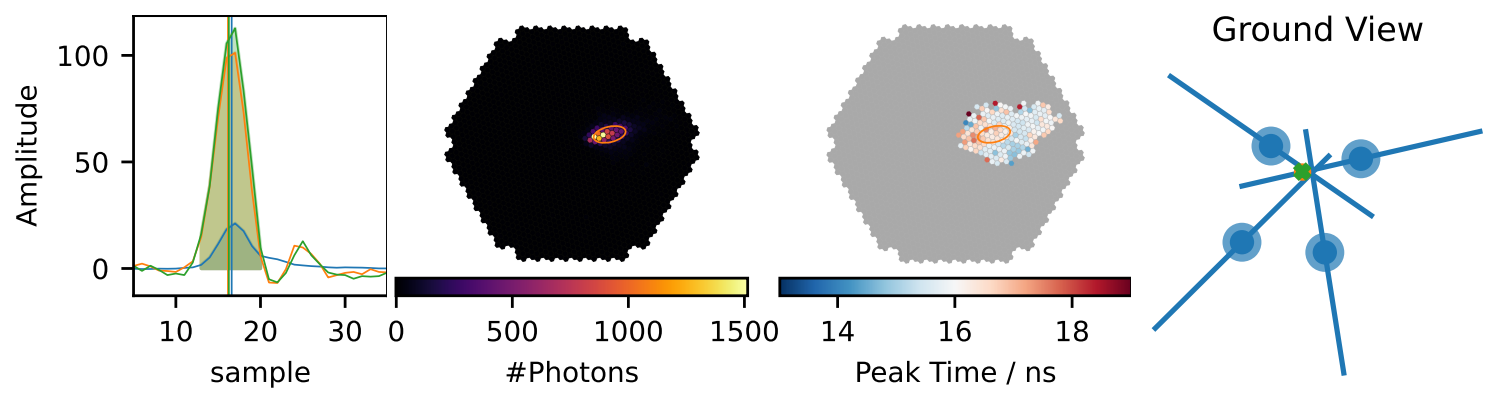

Figure 1: Steps of the analysis up to shower geometry reconstruction: 1. The Cherenkov pulses in each pixel are found and integrated (left) to obtain the number of photons (second from left) and peak times (third from left). 2. The resulting image is cleaned, the pixels not selected are shown in gray in the peak time plot. 3 . The images are parametrized, including the Hillas parameters which are visualized using an ellipse. 4. The physical properties of the primary are reconstructed. The plot on the right shows the impact point of the primary on the ground.

\section{Introduction}

The Cherenkov Telescope Array (CTA ${ }^{1}$ will be the next generation very-high-energy gamma-ray observatory, sensitive to energies between $\sim 20 \mathrm{GeV}$ and $300 \mathrm{TeV}$. It will be composed of over fifty imaging atmospheric Cherenkov telescopes (IACTs) built at two sites to achieve full sky coverage: one on the Canary Island of La Palma, Spain and the other near Paranal, Chile.

CTA will detect gamma rays by measuring the Cherenkov light emitted by extensive air showers, however these are also induced by charged cosmic rays, which form a large background to gammaray observations. The data analysis pipeline of CTA starts with the pre-calibrated raw data from the telescopes in the form of time series data for each pixel and for each telescope that registered a signal from the current shower. The pipeline proceeds to reconstruct the physical properties of the primary particle for each recorded shower, this includes the gamma ray's energy and arrival direction. To remove most of the cosmic-ray induced air showers, also a particle type classification is required.

In the classical analysis approach, the raw data is reduced and aggregated into higher levels of abstraction before finally employing a set of machine learning and geometrical algorithms to reconstruct the physical properties of the primary particle. This is usually performed as a four step procedure, which is currently implemented in ctapipe: image extraction, image cleaning, image parametrization and finally the reconstruction of primary particle properties (see Figure 1). These steps will be detailed in section 2. After reconstruction of the shower events, one last step - based on the pyirf library described in section 3 - selects the best ones on the basis of the specific science case at hand and allows to produce the instrument response functions (IRFs). The pipeline prototype called protopipe and described in section section 4 performs the analysis steps from raw simulated data to IRF production based on both libraries.

\footnotetext{
1 WWW.cta-observatory.org
} 


\section{2. ctapipe}

ctapipe is a python package providing library functions and command-line tools to perform the tasks listed in the previous section. It is developed as open-source software and the project, started in 2015, is hosted on Github. Since then, 26 versions have been released but it is still under heavy development (the latest release at the time of writing is 0.11 .0 [7]). In total, 44 contributors have made this project possible. Releases are published to PyPI and conda packages are provided using conda-forge ${ }^{2}$. ctapipe builds upon the scientific python stack with the main dependencies being astropy [16] for astronomical computations and unit support, numpy [3] and scipy [17] for numerical algorithms and statistics and pytables ${ }^{3}$ for IO using HDF5 ${ }^{4}$. The jit-compiler numba [8] is used to optimize performance-critical parts of the code base.

\subsection{Image Extraction}

The first step in the ctapipe analysis is to reduce the time-series information, i. e. the digitized signals of the Cherenkov photosensors, to the number of photons and their mean arrival time in each pixel. ctapipe supports different algorithms for extracting these quantities from singlepixels waveforms, from simple peak finding algorithms to more complex ones which combine the waveforms of multiple pixels or that fit the expected time evolution of the shower and use that to define the integration window for each pixel.

\subsection{Image Cleaning}

This operation is aimed at identifying pixels which are likely to host real Cherenkov signal. This is usually done by applying a pixel-wise selection via cleaning thresholds based on the photo-electron and peak time values output by the image extraction step. Again, ctapipe supports multiple algorithms to solve this task.

\subsection{Image Parametrization}

After removal of noise pixels, the cleaned image goes through a parametrization in order to make it exploitable by subsequent algorithms, in particular shower geometry reconstructors and/or machinelearning models that assist with with the event property reconstruction. Among the most important parameters are the classical Hillas parameters [5], which describe the orientation and extension of the shower image in the camera, which is needed for the following reconstruction steps. Additionally, ctapipe implements general descriptive statistics of the images, morphological features like the number of isolated pixel groups and parameters describing the containment of the shower's image in each camera.

\subsection{Reconstruction of Event Properties}

While the first three steps can be performed individually for each telescope in the array (monoscopic), this step needs to combine the information from all telescopes to give one common estimate for a recorded shower (stereoscopic).

\footnotetext{
2 conda-forge.org ${ }^{3}$ www.pytables.org ${ }^{4}$ www.hdfgroup.org/solutions/hdf5/
} 
The stereoscopic reconstruction of physical shower parameters can be performed in ctapipe by either of two currently supported approaches: moments-based and template-based.

The moments-based method makes use of a reconstructor which takes as input the parametrized moments of each image (in the default approach the Hillas parameters) from a candidate shower. This input is then combined with a pair-wise geometric reconstruction where each pair of images gets a weight based on the brightness of the images. In case of single, monoscopic telescopes, machine learning can also be used for the reconstruction of the origin, as the geometrical approaches require multiple telescopes.

ctapipe also supports the ImPACT [14] algorithm, an advanced template-based likelihood optimization to reconstruct the event properties, where the expected image for a given set of event properties is calculated from simulations, stored in a database of template images which is then used to perform a likelihood fit to the observed image.

\subsection{Input / Output, visualization and configuration}

IACT events are read from input files using the EventSource interface, which can be implemented for custom file formats using the ctapipe plugin system ${ }^{5}$. There are built-in event source implementations for the simulation file format and ctapipe's own output data format. ctapipe's data model uses its own data structure, called Container, which can be written to and loaded from HDF5 files, supporting transformations and metadata including units.

The ctapipe.visualization module provides classes to display both camera images and telescope array configurations. Two implementations currently exist, one using matplotlib [6] and one using the bokeh ${ }^{6}$ library.

The ctapipe configuration system is build using traitlets ${ }^{7}$, the configuration system developed for IPython. A full configuration tree is built by configurable classes called Components that can include configurable member attributes. Command-line tools use the same configuration mechanism and allow passing configuration for all configurable objects either on the command line or via a configuration file. Many options can be set per telescope type or even per telescope.

\section{Calculating Instrument Response Functions (IRFs) using pyirf}

To be able to estimate physical properties of gamma-ray sources from lists of reconstructed events, the instrumental response to the initial gamma-ray signal must be known. This will depend on the instrument, the specific analysis, environmental conditions and more. In general, the instrumental response of a gamma-ray telescope can be described by the following integral equation, transforming true properties of the gamma rays into the observable quantities:

$$
e(\hat{\alpha}, \hat{\delta}, \hat{E}, t)=\int R(\hat{\alpha}, \hat{\delta}, \hat{E} \mid \alpha, \delta, E, t) \cdot I(\alpha, \delta, E, t) \mathrm{d} \Omega \mathrm{d} E+b(\hat{\alpha}, \hat{\delta}, \hat{E})
$$

Where $\alpha, \delta$ and $E$ are the right ascension, declination of the gamma ray origin and its total energy, while $\hat{\alpha}, \hat{\delta}, \hat{E}$ are the corresponding reconstructed quantities obtained from the analysis pipeline. $I$ is the source term, the true gamma-ray signal arriving at earth at the given position, energy and time $t$. $R$ is the IRF, the convolution kernel translating true quantities to the observed ones, $b$ is

\footnotetext{
5 E.g.: github.com/cta-observatory/ctapipe_io_lst 6 bokeh.org/ 7 traitlets.readthedocs.io/
} 
the irreducible background and $e$ is the expected event distribution as measured by the experiment. The solid angle integration over $\alpha, \delta$ is denoted using $\mathrm{d} \Omega$.

The IRF can only be estimated from labeled data, where the true and reconstructed quantities are both known. In the case of CTA these labeled datasets are created via Monte Carlo simulations using CORSIKA [4] to simulate the extensive air showers, followed by the detector simulation performed by sim_telarray [1].

In classical IACT analysis, the IRF is factorized into three independent components, making the strong assumption that the migrations between the different observables are statistically independent. This factorization yields:

$$
R(\hat{\alpha}, \hat{\delta}, \hat{E} \mid \alpha, \delta, E, t)=A_{\mathrm{eff}}(\alpha, \delta, E, t) \cdot \operatorname{PSF}(\hat{\alpha}, \hat{\delta} \mid \alpha, \delta, E, t) \cdot D(\hat{E} \mid \alpha, \delta, E, t)
$$

Where $A_{\text {eff }}$ is the effective area, the detection probability times the observed area for a gamma ray with given true properties, PSF is the point spread function, i. e. the convolution kernel for the reconstructed gamma-ray origin and $D$ is the energy dispersion, the migration between true energy $E$ and reconstructed energy $\hat{E}$. Instead of continuous functions, these IRFs are calculated and stored as binned quantities filled from simulated events.

pyirf is a python library for calculating these IRFs from labeled, reconstructed event lists as created by the event reconstruction pipeline. The latest version of pyirf at the time of writing is v0 . 5 . 0 [12], which supports calculating most IRFs formats defined in Gamma-Astro-Data-Formats (GADF, [2]) and can export these into the FITS-based data format defined therein. Additionally, pyirf contains functionality to calculate flux sensitivity of gamma-ray instruments according to the requirements laid out for CTA and the optimization of event selection criteria to obtain the best flux sensitivity.

\section{4. protopipe}

protopipe is a pipeline prototype for CTA based on the ctapipe and pyirf libraries. It is distributed as a python package on the PyPI platform; the latest release at the time of writing is 0.4.0.post1 [15]. Started as an independent project for image cleaning studies by the CTA Consortium group at CEA-Saclay/IRFU, it has been developed as an open-source package for the whole consortium since September 2019. Since then, its development has been steered by the will to substitute the historical pipelines currently in use for the production of the official IRFs for CTA. Such pipelines have been inherited from the VERITAS (EventDisplay [10]) and MAGIC (MARS [19]) experiments and adapted to the CTA scenario by their maintainers. Even if they provide satisfactory results, they are not in line with the software requirements of CTA and not easily exploitable by the whole consortium. The development of protopipe is strongly influenced by a step-by-step comparison with such pipelines, which translates in a continuous code migration into the ctapipe and pyirf libraries (algorithms and support of additional analysis operations).

protopipe has been built around the two libraries described in this work by constantly trying to support their latest stable releases. It also provides a module for multivariate analysis using supervised machine-learning techniques (protopipe.mva), used to reconstruct energy and particle type of the events. 
The pipeline provides four tools based on ctapipe, protopipe.mva, ctapipe and pyirf respectively. Each tool is a python executable configurable via YAML-based configuration files. protopipe also provides a way to launch the analysis on computing grids featuring the DIRAC interware ${ }^{8}$. The tools can be launched on the grid thanks to an interface code developed separately from the main package and based on CTADIRAC ${ }^{9}$, a version of the DIRAC middleware customized for CTA.

\subsection{Description of the pipeline workflow}

A full dataset composed of simulated events from primary gammas, protons and electrons is split at the beginning of the analysis in sub-datasets. Depending on the workflow of choice, a step of the pipeline will correspond to a tool being applied to one or more sub-datasets. The currently tested workflow is the following:

- part of the gamma rays are used to train an energy reconstruction model,

- part of the gamma rays and part of the protons are used to train a particle classification model (making use also of the reconstructed energy),

- the remaining gamma rays and protons together with the full electron dataset are fully analyzed.

In a real scenario of a gamma-ray analysis, the entire third sub-dataset and the proton subdataset used to train the particle classifier would correspond to data observed by the telescope array. An overview of the workflow is shown in Figure 2 and the tools are defined in the following sections.

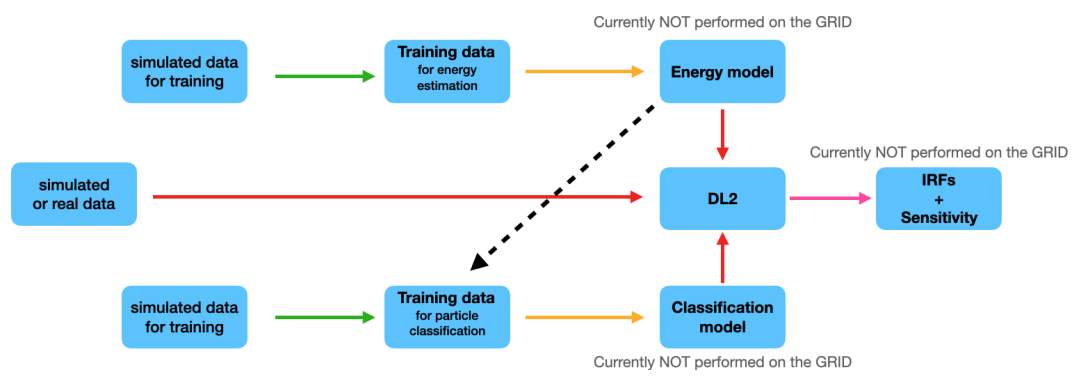

Figure 2: Current pipeline workflow tested on full-scale analyses on the GRID. The actions performed by the tools 1, 2, 3 and 4 are highlighted by green, orange, red and pink arrows respectively. Reconstructed energy is used as a model feature when training particle classification (black dashed arrow)

\subsubsection{Tool 1: preparation of training data}

This tool is based on ctapipe and it produces data in a format suitable for model training. This format is a combination of data levels as defined by the data models in ctapipe: DL1b (image parameters) and part of DL2 (reconstructed shower geometry). The transformation of raw data into DL1b data makes use of the library capabilities described in paragraphs 2.1 to 2.3. Since the pipeline workflow currently tested (see Fig.2) comprises the use of two models (one for energy reconstruction and the other for particle classification) this tool is used in two separate steps of that analysis.

\footnotetext{
8 dirac.readthedocs.io/en/latest/ 9 github.com/cta-observatory/CTADIRAC
} 


\subsubsection{Tool 2: production of models}

The production of machine-learning models is performed by the tool based on the protopipe .mva module. The dependencies are few: numpy and pandas to deal internally with tables of data, joblib for I/O support and scikit-learn to create the models and fit the test data. The models currently tested are part of the sklearn.ensemble module: AdaBoostRegressor or RandomForestRegressor for energy reconstruction and RandomForestClassifier for particle classification. It is possible to perform tuning of the hyper-parameters via an exhaustive search over lists of parameter values specified by the user (sklearn.model_selection.GridSearchCV). The tool outputs both model and tables of the events selected for training and testing as gzip-compressed pickled objects.

\subsubsection{Tool 3: production of fully-analyzed events}

This tool performs the full reconstruction pipeline and is applied to events which have to be independent from those used by the previous tools. The operations performed are those described by paragraphs 2.1 to 2.4. In particular the tool requires as an input the models produced by Tool 2 in order to reconstruct both energy and particle type. The models' input file format currently supported is the one output by the Tool 2 .

\subsubsection{Tool 4: production of IRFs and optimized cuts}

This tool is based on the functions provided by the pyirf library and performs the following sequence of operations:

1. find the best cutoff in gammaness score, which is the result of the particle type classification, to best discriminate between signal and background, as well as the angular cut to obtain the best sensitivity for a given amount of observation time and a given template for the source of interest,

2. estimate the sensitivity from the optimized cuts,

3. compute the IRFs from the same selected events.

The current output format is the one supported by pyirf: it builds on the data format specification given by the GADF integrated by input coming from CTA optimizations.

\section{Conclusions and Outlook}

ctapipe and pyirf offer open-source tools to solve a critical part of the analysis of IACT data. Using the IO plugin system, ctapipe can be used to process data by all experiments, see for example [13] for a combined analysis of LST-1 and MAGIC observations. While the current version of ctapipe performs event property estimation using geometrical or template based algorithms, the use of modern machine learning techniques is also investigated (see for example [18] and [11]). Performance of an analysis using ctapipe and pyirf on simulated data and first results on data from observations performed by the LST-1 are reported in [9]. protopipe is being currently developed with the goal of superseding the reference analyses for the planned arrays, currently performed by EventDisplay and MARS. It takes into account all supported cameras, optics and array configurations for CTA, enabling a high degree of flexibility to accommodate diverse instrument 
configurations. It will be used to produce sets of IRFs sufficiently large to describe and investigate the performance of CTA under any required observing condition and science case and to analyze data from the whole set of telescopes.

Acknowledgements: We gratefully acknowledge financial support from the agencies and organizations listed here: www.cta-observatory .org/consortium_acknowledgments

\section{References}

[1] K. Bernlöhr. "Simulation of imaging atmospheric Cherenkov telescopes with CORSIKA and sim_telarray." In: Astroparticle Physics 30.3 (Oct. 2008), pp. 149-158. DoI: 10.1016/j . astropartphys. 2008.07.009.

[2] C. Deil et al. Data formats for gamma-ray astronomy - version 0.2. 2018. Dor: 10.5281/zenodo. 1409831.

[3] C. R. Harris et al. “Array programming with NumPy.” In: Nature 585 (2020), pp. 357-362. Dor: 10.1038 / s41586-020-2649-2.

[4] D. Heck et al. CORSIKA: A Monte Carlo Code to Simulate Extensive Air Showers. Tech. rep. FZKA 6019. Forschungszentrum Karlsruhe, 1998.

[5] A. M. Hillas. "Cerenkov light images of EAS produced by primary gamma." In: Proceedings, 19th International Cosmic Ray Conference. Vol. 3. 1985.

[6] J. D. Hunter. "Matplotlib: A 2D graphics environment." In: Computing in Science \& Engineering 9.3 (2007), pp. 90-95. DOI: 10.1109/MCSE. 2007.55.

[7] K. Kosack et al. ctapipe. Version v0.10.5. Mar. 2021. DoI: 10 . 5281/zenodo . 4581045. URL: https : // github.com/cta-observatory/ctapipe.

[8] S. K. Lam, A. Pitrou, and S. Seibert. "Numba: A llvm-based python jit compiler.” In: Proceedings of the Second Workshop on the LLVM Compiler Infrastructure in HPC. 2015, pp. 1-6.

[9] R. López-Coto et al. for the CTA LST Project. "Physics Performance of the Large Size Telescope1prototype of the Cherenkov Telescope Array.” In: these proceedings. 806. 2021. Dor: 10.22323/1.395.0806.

[10] G. Maier and J. Holder. "Eventdisplay. An analysis and reconstruction package for ground-based gammaray astronomy." In: Proceedings, $35^{\text {th }}$ International Cosmic Ray Conferenc. 747. Busan, Korea, 2017. DoI: 10.22323/1.301.0747.

[11] T. Miener et al. for the CTA Consortium. "Reconstruction of stereoscopic CTA events using deep learning with CTLearn." In: these proceedings. 730. 2021. DOI: 10.22323/1.395.0730.

[12] M. Nöthe et al. pyirf. Version v0.5.0. May 2021. Dor: 10.5281/zenodo . 4748994. uRL: https: //github . com/cta-observatory/pyirf.

[13] Y. Ohtani et al. for the CTA LST Project and MAGIC Collaboration. "Cross-calibration and combined analysis of the CTA-LST prototype and the MAGIC telescopes." In: these proceedings. 724. 2021. Dor: 10.22323/1. 395.0724 .

[14] R. D. Parsons and J. A. Hinton. "A Monte Carlo template based analysis for air-Cherenkov arrays." In: Astroparticle Physics 56 (2014), pp. 26-34. DoI: 10.1016/j . astropartphys.2014.03.002.

[15] M. Peresano et al. protopipe. DoI: 10 . 5281 / zenodo . 4586754. urL: https : / / github . com / cta observatory/protopipe.

[16] A. M. Price-Whelan et al. for the Astropy Collaboration. "The Astropy Project. Building an Open-science Project and Status of the v2.0 Core Package.” In: The Astronomical Journal 156, 123 (Sept. 2018). Dor: 10 . 3847/1538$3881 /$ aabc4f.

[17] P. Virtanen et al. "SciPy 1.0: Fundamental Algorithms for Scientific Computing in Python.” In: Nature Methods 17 (2020), pp. 261-272. DoI: 10.1038/s41592-019-0686-2. 
[18] T. Vuillaume et al. for the CTA LST Project. "Analysis of the Cherenkov Telescope Array first Large Size Telescope real data using convolutional neural networks.” In: these proceedings. 703. 2021. Dor: 10.22323/1. 395.0703 .

[19] R. Zanin et al. for the MAGIC Collaboration. "MARS, the MAGIC analysis and reconstruction software." In: Proceedings, $33^{\text {rd }}$ International Cosmic Ray Conference. Rio de Janeiro, Brazil, 2013.

\section{Full Authors List: CTA Consortium}

H. Abdalla ${ }^{1}$, H. Abe ${ }^{2}$, S. Abe ${ }^{2}$, A. Abusleme ${ }^{3}$, F. Acero ${ }^{4}$, A. Acharyya ${ }^{5}$, V. Acín Portella ${ }^{6}$, K. Ackley $^{7}$, R. Adam ${ }^{8}$, C. Adams $^{9}$, S.S. Adhikari ${ }^{10}$, I. Aguado-Ruesga ${ }^{11}$, I. Agudo ${ }^{12}$, R. Aguilera ${ }^{13}$, A. Aguirre-Santaella ${ }^{14}$, F. Aharonian ${ }^{15}$, A. Alberdi ${ }^{12}$, R. Alfaro ${ }^{16}$, J. Alfaro ${ }^{3}$, C. Alispach ${ }^{17}$, R. Aloisio ${ }^{18}$, R. Alves Batista ${ }^{19}$, J.-P. Amans ${ }^{20}$, L. Amati ${ }^{21}$, E. Amato ${ }^{22}$, L. Ambrogi ${ }^{18}$, G. Ambrosi ${ }^{23}$, M. Ambrosio ${ }^{24}$, R. Ammendola ${ }^{25}$, J. Anderson ${ }^{26}$, M. Anduze ${ }^{8}$, E.O. Angüner ${ }^{27}$, L.A. Antonelli ${ }^{28}$, V. Antonuccio ${ }^{29}$, P. Antoranz ${ }^{30}$,

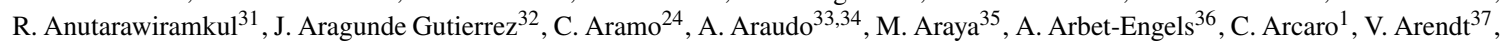
C. Armand ${ }^{38}$, T. Armstrong ${ }^{27}$, F. Arqueros ${ }^{11}$, L. Arrabito ${ }^{39}$, B. Arsioli ${ }^{40}$, M. Artero ${ }^{41}$, K. Asano $^{2}$, Y. Ascasíbar ${ }^{14}$, J. Aschersleben ${ }^{42}$, M. Ashley ${ }^{43}$, P. Attinà ${ }^{44}$, P. Aubert ${ }^{45}$, C. B. Singh ${ }^{19}$, D. Baack ${ }^{46}$, A. Babic ${ }^{47}$, M. Backes ${ }^{48}$, V. Baena ${ }^{13}$, S. Bajtlik ${ }^{49}$, A. Baktash $^{50}$, C. Balazs ${ }^{7}$, M. Balbo ${ }^{38}$, O. Ballester ${ }^{41}$, J. Ballet ${ }^{4}$, B. Balmaverde ${ }^{44}$, A. Bamba ${ }^{51}$, R. Bandiera ${ }^{22}$, A. Baquero Larriva ${ }^{11}$, P. Barai ${ }^{19}$, C. Barbier ${ }^{45}$, V. Barbosa Martins ${ }^{52}$, M. Barcelo ${ }^{53}$, M. Barkov ${ }^{54}$, M. Barnard ${ }^{1}$, L. Baroncelli ${ }^{21}$, U. Barres de Almeida ${ }^{40}$, J.A. Barrio ${ }^{11}$, D. Bastieri ${ }^{55}$, P.I. Batista ${ }^{52}$, I. Batkovic ${ }^{55}$, C. Bauer ${ }^{53}$, R. Bautista-González ${ }^{56}$, J. Baxter ${ }^{2}$, U. Becciani2 ${ }^{29}$, J. Becerra González ${ }^{32}$, Y. Becherini ${ }^{57}$, G. Beck ${ }^{58}$, J. Becker Tjus ${ }^{59}$, W. Bednarek ${ }^{60}$, A. Belfiore ${ }^{61}$, L. Bellizzi ${ }^{62}$, R. Belmont ${ }^{4}$, W. Benbow ${ }^{63}$, D. Berge ${ }^{52}$, E. Bernardini ${ }^{52}$, M.I. Bernardos ${ }^{55}$, K. Bernlöhr ${ }^{53}$, A. Berti ${ }^{64}$, M. Berton ${ }^{65}$, B. Bertucci ${ }^{23}$, V. Beshley ${ }^{66}$, N. Bhatt ${ }^{67}$, S. Bhattacharyya ${ }^{67}$,

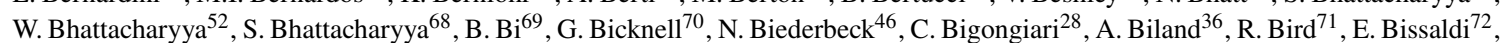
J. Biteau ${ }^{73}$, M. Bitossi ${ }^{74}$, O. Blanch ${ }^{41}$, M. Blank ${ }^{50}$, J. Blazek ${ }^{33}$, J. Bobin ${ }^{75}$, C. Boccato ${ }^{76}$, F. Bocchino ${ }^{77}$, C. Boehm ${ }^{78}$, M. Bohacova ${ }^{33}$, C. Boisson ${ }^{20}$, J. Boix ${ }^{41}$, J.-P. Bolle ${ }^{52}$, J. Bolmont ${ }^{79}$, G. Bonanno ${ }^{29}$, C. Bonavolontă ${ }^{24}$, L. Bonneau Arbeletche ${ }^{80}$, G. Bonnoli ${ }^{12}$, P. Bordas ${ }^{81}$, J. Borkowski ${ }^{49}$, S. Bórquez ${ }^{35}$, R. Bose ${ }^{82}$, D. Bose ${ }^{83}$, Z. Bosnjak ${ }^{47}$, E. Bottacini ${ }^{55}$, M. Böttcher ${ }^{1}$, M.T. Botticella ${ }^{84}$, C. Boutonnet ${ }^{85}$, F. Bouyjou ${ }^{75}$, V. Bozhilov ${ }^{86}$, E. Bozzo ${ }^{38}$, L. Brahimi ${ }^{39}$, C. Braiding ${ }^{43}$, S. Brau-Nogué ${ }^{87}$, S. Breen ${ }^{78}$, J. Bregeon ${ }^{39}$, M. Breuhaus ${ }^{53}$, A. Brill ${ }^{9}$, W. Brisken ${ }^{88}$, E. Brocato ${ }^{28}$, A.M. Brown ${ }^{5}$, K. Brügge ${ }^{46}$, P. Brun ${ }^{89}$, P. Brun ${ }^{39}$, F. Brun ${ }^{89}$, L. Brunetti ${ }^{45}$, G. Brunetti ${ }^{90}$, P. Bruno ${ }^{29}$, A. Bruno ${ }^{91}$, A. Bruzzese ${ }^{6}$, N. Bucciantini ${ }^{22}$, J. Buckley ${ }^{82}$, R. Bühler ${ }^{52}$, A. Bulgarelli' ${ }^{21}$, T. Bulik ${ }^{92}$, M. Bünning ${ }^{52}$, M. Bunse ${ }^{46}$, M. Burton ${ }^{93}$, A. Burtovoi ${ }^{76}$, M. Buscemi ${ }^{94}$, S. Buschjäger ${ }^{46}$, G. Busetto ${ }^{55}$, J. Buss ${ }^{46}$, K. Byrum ${ }^{26}$, A. Caccianiga ${ }^{95}$, F. Cadoux ${ }^{17}$, A. Calanducci ${ }^{29}$, C. Calderón ${ }^{3}$, J. Calvo $\operatorname{Tovar}^{32}$, R. Cameron ${ }^{96}$, P. Campaña ${ }^{35}$, R. Canestrari ${ }^{91}$, F. Cangemi ${ }^{79}$, B. Cantlay ${ }^{31}$, M. Capalbi ${ }^{91}$, M. Capasso ${ }^{9}$, M. Cappi ${ }^{21}$, A. Caproni ${ }^{97}$, R. Capuzzo-Dolcetta ${ }^{28}$, P. Caraveo ${ }^{61}$, V. Cárdenas ${ }^{98}$, L. Cardiel ${ }^{41}$, M. Cardillo ${ }^{99}$, C. Carlile ${ }^{100}$, S. Caroff ${ }^{45}$, R. Carosi ${ }^{74}$, A. Carosi ${ }^{17}$, E. Carquín ${ }^{35}$, M. Carrère ${ }^{39}$, J.-M. Casandjian ${ }^{4}$, S. Casanova ${ }^{101,53}$, E. Cascone ${ }^{84}$, F. Cassol ${ }^{27}$, A.J. Castro-Tirado ${ }^{12}$, F. Catalani ${ }^{102}$, O. Catalano ${ }^{91}$, D. Cauz ${ }^{103}$, A. Ceccanti6 ${ }^{64}$, C. Celestino Silva ${ }^{80}$, S. Celli ${ }^{18}$, K. Cerny ${ }^{104}$, M. Cerruti ${ }^{85}$, E. Chabanne ${ }^{45}$, P. Chadwick ${ }^{5}$, Y. Chai ${ }^{105}$, P. Chambery ${ }^{106}$, C. Champion ${ }^{85}$, S. Chandra ${ }^{1}$, S. Chaty ${ }^{4}$, A. Chen ${ }^{58}$, K. Cheng ${ }^{2}$, M. Chernyakova ${ }^{107}$, G. Chiaro ${ }^{61}$, A. Chiavassa ${ }^{64,108}$, M. Chikawa ${ }^{2}$, V.R. Chitnis ${ }^{109}$, J. Chudoba ${ }^{33}$, L. Chytka ${ }^{104}$, S. Cikota ${ }^{47}$, A. Circiello ${ }^{24,110}$, P. Clark ${ }^{5}$, M. Çolak ${ }^{41}$, E. Colombo ${ }^{32}$, J. Colome ${ }^{13}$, S. Colonges ${ }^{85}$, A. Comastri ${ }^{21}$, A. Compagnino ${ }^{91}$, V. Conforti ${ }^{21}$, E. Congiu ${ }^{95}$, R. Coniglione ${ }^{94}$, J. Conrad ${ }^{111}$, F. Conte ${ }^{53}$, J.L. Contreras ${ }^{11}$, P. Coppi ${ }^{112}$, R. Cornat ${ }^{8}$, J. Coronado-Blazquez ${ }^{14}$, J. Cortina ${ }^{113}$, A. Costa ${ }^{29}$, H. Costantini ${ }^{27}$, G. Cotter ${ }^{114}$, B. Courty ${ }^{85}$, S. Covino ${ }^{95}$, S. Crestan ${ }^{61}$,

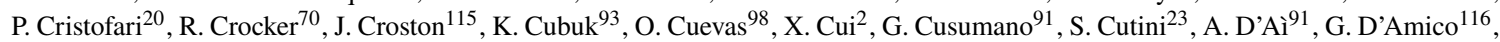
F. D'Ammando ${ }^{90}$, P. D’Avanzo ${ }^{95}$, P. Da Vela ${ }^{74}$, M. Dadina ${ }^{21}$, S. Dai ${ }^{117}$, M. Dalchenko ${ }^{17}$, M. Dall' Ora ${ }^{84}$, M.K. Daniel ${ }^{63}$, J. Dauguet ${ }^{85}$, I. Davids ${ }^{48}$, J. Davies ${ }^{114}$, B. Dawson ${ }^{118}$, A. De Angelis ${ }^{55}$, A.E. de Araújo Carvalho ${ }^{40}$, M. de Bony de Lavergne ${ }^{45}$, V. De Caprio ${ }^{84}$, G. De Cesare ${ }^{21}$, F. De Frondat ${ }^{20}$, E.M. de Gouveia Dal Pino ${ }^{19}$, I. de la Calle ${ }^{11}$, B. De Lotto ${ }^{103}$, A. De Luca ${ }^{61}$, D. De Martino ${ }^{84}$, R.M. de Menezes $^{19}$, M. de Naurois ${ }^{8}$, E. de Oña Wilhelmi ${ }^{13}$, F. De Palma ${ }^{64}$, F. De Persio ${ }^{119}$, N. de Simone ${ }^{52}$, V. de Souza ${ }^{80}$, M. Del Santo ${ }^{91}$, M.V. del Valle ${ }^{19}$, E. Delagnes ${ }^{75}$, G. Deleglise ${ }^{45}$, M. Delfino Reznicek ${ }^{6}$, C. Delgado ${ }^{113}$, A.G. Delgado Giler ${ }^{80}$, J. Delgado Mengual ${ }^{6}$, R. Della Ceca ${ }^{95}$, M. Della Valle ${ }^{84}$, D. della Volpe ${ }^{17}$, D. Depaoli ${ }^{64,108}$, D. Depouez ${ }^{27}$, J. Devin ${ }^{85}$, T. Di Girolamo ${ }^{24,110}$, C. Di Giulio ${ }^{25}$, A. Di Piano ${ }^{21}$, F. Di Pierro ${ }^{64}$, L. Di Venere ${ }^{120}$, C. Díaz ${ }^{113}$, C. Díaz-Bahamondes ${ }^{3}$, C. Dib ${ }^{35}$, S. Diebold $^{69}$, S. Digel ${ }^{96}$, R. Dima $^{55}$, A. Djannati-Ataï ${ }^{85}$, J. Djuvsland ${ }^{16}$, A. Dmytriiev ${ }^{20}$, K. Docher ${ }^{9}$, A. Domínguez ${ }^{11}$, D. Dominis Prester ${ }^{121}$, A. Donath ${ }^{53}$, A. Donini ${ }^{41}$, D. Dorner ${ }^{122}$, M. Doro ${ }^{55}$, R.d.C. dos Anjos ${ }^{123}$, J.-L. Dournaux ${ }^{20}$, T. Downes ${ }^{107}$, G. Drake ${ }^{26}$, H. Drass ${ }^{3}$, D. Dravins ${ }^{100}$, C. Duangchan ${ }^{31}$,

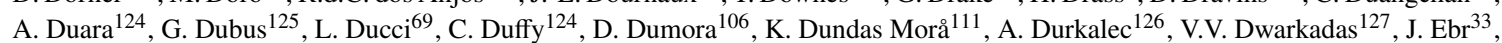
C. Eckner ${ }^{45}$, J. Eder ${ }^{105}$, A. Ederoclite ${ }^{19}$, E. Edy ${ }^{8}$, K. Egberts ${ }^{128}$, S. Einecke ${ }^{118}$, J. Eisch ${ }^{129}$, C. Eleftheriadis ${ }^{130}$, D. Elsässer ${ }^{46}$, G. Emery ${ }^{17}$, D. Emmanoulopoulos ${ }^{115}$, J.-P. Ernenwein ${ }^{27}$, M. Errando ${ }^{82}$, P. Escarate ${ }^{35}$, J. Escudero ${ }^{12}$, C. Espinoza ${ }^{3}$, S. Ettori ${ }^{21}$, A. Eungwanichayapant ${ }^{31}$, P. Evans ${ }^{124}$, C. Evoli ${ }^{18}$, M. Fairbairn ${ }^{131}$, D. Falceta-Goncalves ${ }^{132}$, A. Falcone ${ }^{133}$, V. Fallah Ramazani ${ }^{65}$, R. Falomo ${ }^{76}$, K. Farakos ${ }^{134}$, G. Fasola ${ }^{20}$, A. Fattorini ${ }^{46}$, Y. Favre ${ }^{17}$, R. Fedora ${ }^{135}$, E. Fedorova ${ }^{136}$, S. Fegan ${ }^{8}$, K. Feijen ${ }^{118}$, Q. Feng ${ }^{9}$, G. Ferrand ${ }^{54}$, G. Ferrara ${ }^{94}$, O. Ferreira ${ }^{8}$, M. Fesquet ${ }^{75}$, E. Fiandrini ${ }^{23}$, A. Fiasson ${ }^{45}$, M. Filipovic ${ }^{117}$, D. Fink ${ }^{105}$, J.P. Finley ${ }^{137}$, V. Fioretti ${ }^{21}$, D.F.G. Fiorillo ${ }^{24,110}$, M. Fiorini ${ }^{61}$, S. Flis ${ }^{52}$, H. Flores ${ }^{20}$, L. Foffano ${ }^{17}$, C. Föhr ${ }^{33}$, M.V. Fonseca ${ }^{11}$, L. Font ${ }^{138}$, G. Fontaine ${ }^{8}$, O. Fornieri ${ }^{52}$, P. Fortin ${ }^{63}$, L. Fortson ${ }^{88}$, N. Fouque ${ }^{45}$, A. Fournier ${ }^{106}$, B. Fraga ${ }^{40}$, A. Franceschini ${ }^{76}$, F.J. Franco ${ }^{30}$, A. Franco Ordovas $^{32}$, L. Freixas Coromina ${ }^{113}$, L. Fresnillo ${ }^{30}$, C. Fruck ${ }^{105}$, D. Fugazza ${ }^{95}$, Y. Fujikawa ${ }^{139}$, Y. Fujita ${ }^{2}$, S. Fukami ${ }^{2}$, Y. Fukazawa $^{140}$, Y. Fukui ${ }^{141}$, D. Fulla ${ }^{52}$, S. Funk ${ }^{142}$, A. Furniss ${ }^{143}$, O. Gabella ${ }^{39}$, S. Gabici ${ }^{85}$, D. Gaggero ${ }^{14}$, G. Galanti $^{61}$, G. Galaz $^{3}$, P. Galdemard ${ }^{144}$, Y. Gallant ${ }^{39}$, D. Galloway ${ }^{7}$, S. Gallozzi ${ }^{28}$, V. Gammaldi ${ }^{14}$, R. Garcia ${ }^{41}$, E. Garcia ${ }^{45}$, E. García ${ }^{13}$, R. Garcia López ${ }^{32}$, M. Garczarczyk ${ }^{52}$, F. Gargano ${ }^{120}$, C. Gargano ${ }^{11}$, S. Garozzo ${ }^{29}$, D. Gascon ${ }^{81}$, T. Gasparetto ${ }^{145}$, D. Gasparrini ${ }^{25}$, H. Gasparyan ${ }^{52}$, 
M. Gaug ${ }^{138}$, N. Geffroy ${ }^{45}$, A. Gent ${ }^{146}$, S. Germani ${ }^{76}$, L. Gesa ${ }^{13}$, A. Ghalumyan ${ }^{147}$, A. Ghedina ${ }^{148}$, G. Ghirlanda ${ }^{95}$, F. Gianotti ${ }^{21}$, S. Giarrusso ${ }^{91}$, M. Giarrusso ${ }^{94}$, G. Giavitto $^{52}$, B. Giebels ${ }^{8}$, N. Giglietto ${ }^{72}$, V. Gika ${ }^{134}$, F. Gillardo $^{45}$, R. Gimenes ${ }^{19}$, F. Giordano $^{149}$, G. Giovannini ${ }^{90}$, E. Giro ${ }^{76}$, M. Giroletti ${ }^{90}$, A. Giuliani ${ }^{61}$, L. Giunti ${ }^{85}$, M. Gjaja ${ }^{9}$, J.-F. Glicenstein ${ }^{89}$, P. Gliwny ${ }^{60}$, N. Godinovic ${ }^{150}$, H. Göksu ${ }^{53}$, P. Goldoni ${ }^{85}$, J.L. Gómez ${ }^{12}$, G. Gómez-Vargas ${ }^{3}$, M.M. González ${ }^{16}$, J.M. González ${ }^{151}$, K.S. Gothe ${ }^{109}$, D. Götz ${ }^{4}$, J. Goulart Coelho $^{123}$, K. Gourgouliatos 5 , T. Grabarczyk ${ }^{152}$, R. Graciani ${ }^{81}$, P. Grandi ${ }^{21}$, G. Grasseau ${ }^{8}$, D. Grasso ${ }^{74}$, A.J. Green ${ }^{78}$, D. Green ${ }^{105}$, J. Green ${ }^{28}$, T. Greenshaw ${ }^{153}$, I. Grenier ${ }^{4}$, P. Grespan ${ }^{55}$, A. Grillo ${ }^{29}$, M.-H. Grondin ${ }^{106}$, J. Grube ${ }^{131}$, V. Guarino ${ }^{26}$, B. Guest ${ }^{37}$, O. Gueta ${ }^{52}$, M. Gündüz ${ }^{59}$, S. Gunji ${ }^{154}$, A. Gusdorf ${ }^{20}$, G. Gyuk ${ }^{155}$, J. Hackfeld ${ }^{59}$, D. Hadasch ${ }^{2}$, J. Haga ${ }^{139}$, L. Hagge ${ }^{52}$, A. Hahn ${ }^{105}$,

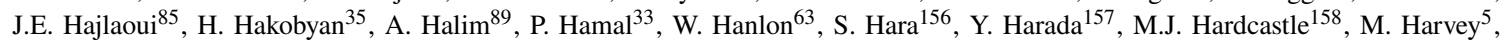
K. Hashiyama ${ }^{2}$, T. Hassan Collado ${ }^{113}$, T. Haubold ${ }^{105}$, A. Haupt ${ }^{52}$, U.A. Hautmann ${ }^{159}$, M. Havelka ${ }^{33}$, K. Hayashi ${ }^{141}$, K. Hayashi ${ }^{160}$, M. Hayashida ${ }^{161}, \mathrm{H} . \mathrm{He}^{54}$, L. Heckmann ${ }^{105}$, M. Heller ${ }^{17}$, J.C. Helo ${ }^{35}$, F. Henault ${ }^{125}$, G. Henri ${ }^{125}$, G. Hermann ${ }^{53}$, R. Hermel ${ }^{45}$, S. Hernández Cadena ${ }^{16}$, J. Herrera Llorente ${ }^{32}$, A. Herrero ${ }^{32}$, O. Hervet ${ }^{143}$, J. Hinton ${ }^{53}$, A. Hiramatsu ${ }^{157}$, N. Hiroshima ${ }^{54}$, K. Hirotani ${ }^{2}$, B. Hnatyk ${ }^{136}$, R. Hnatyk ${ }^{136}$, J.K. Hoang ${ }^{11}$, D. Hoffmann ${ }^{27}$, W. Hofmann ${ }^{53}$, C. Hoischen ${ }^{128}$, J. Holder ${ }^{162}$, M. Holler ${ }^{163}$, B. Hona ${ }^{164}$,

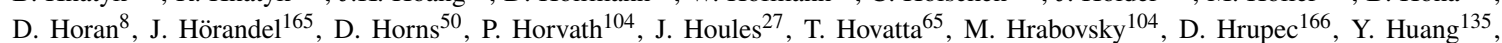
J.-M. Huet ${ }^{20}$, G. Hughes ${ }^{159}$, D. Hui ${ }^{2}$, G. Hull ${ }^{73}$, T.B. Humensky ${ }^{9}$, M. Hütten ${ }^{105}$, R. Iaria ${ }^{77}$, M. Iarlori ${ }^{18}$, J.M. Illa ${ }^{41}$, R. Imazawa ${ }^{140}$, D. Impiombato ${ }^{91}$, T. Inada ${ }^{2}$, F. Incardona ${ }^{29}$, A. Ingallinera ${ }^{29}$, Y. Inome ${ }^{2}$, S. Inoue ${ }^{54}$, T. Inoue ${ }^{141}$, Y. Inoue ${ }^{167}$, A. Insolia ${ }^{120,94}$, F. Iocco ${ }^{24,110}$, K. Ioka ${ }^{168}$, M. Ionica ${ }^{23}$, M. Iori ${ }^{119}$, S. Iovenitti ${ }^{95}$, A. Iriarte ${ }^{16}$, K. Ishio ${ }^{105}$, W. Ishizaki ${ }^{168}$, Y. Iwamura ${ }^{2}$, C. Jablonski ${ }^{105}$, J. Jacquemier ${ }^{45}$, M. Jacquemont ${ }^{45}$, M. Jamrozy ${ }^{169}$, P. Janecek ${ }^{33}$, F. Jankowsky ${ }^{170}$, A. Jardin-Blicq ${ }^{31}$, C. Jarnot ${ }^{87}$, P. Jean ${ }^{87}$, I. Jiménez Martínez $^{113}$, W. Jin ${ }^{171}$, L. Jocou ${ }^{125}$, N. Jordana ${ }^{172}$, M. Josselin ${ }^{73}$, L. Jouvin ${ }^{41}$, I. Jung-Richardt ${ }^{142}$, F.J.P.A. Junqueira ${ }^{19}$, C. JuramyGilles $^{79}$, J. Jurysek ${ }^{38}$, P. Kaaret ${ }^{173}$, L.H.S. Kadowaki ${ }^{19}$, M. Kagaya ${ }^{2}$, O. Kalekin ${ }^{142}$, R. Kankanyan ${ }^{53}$, D. Kantzas ${ }^{174}$, V. Karas ${ }^{34}$, A. Karastergiou ${ }^{114}$, S. Karkar ${ }^{79}$, E. Kasai ${ }^{48}$, J. Kasperek ${ }^{175}$, H. Katagiri ${ }^{176}$, J. Kataoka ${ }^{177}$, K. Katarzyński ${ }^{178}$, S. Katsuda ${ }^{179}$, U. Katz ${ }^{142}$, N. Kawanaka ${ }^{180}$, D. Kazanas ${ }^{130}$, D. Kerszberg ${ }^{41}$, B. Khélifi ${ }^{85}$, M.C. Kherlakian ${ }^{52}$, T.P. Kian ${ }^{181}$, D.B. Kieda ${ }^{164}$, T. Kihm ${ }^{53}$, S. Kim ${ }^{3}$, S. Kimeswenger ${ }^{163}$, S. Kisaka ${ }^{140}$, R. Kissmann ${ }^{163}$, R. Kleijwegt ${ }^{135}$, T. Kleiner ${ }^{52}$, G. Kluge ${ }^{10}$, W. Kluźniak ${ }^{49}$, J. Knapp ${ }^{52}$, J. Knödlseder ${ }^{87}$, A. Kobakhidze ${ }^{78}$, Y. Kobayashi ${ }^{2}$, B. Koch ${ }^{3}$, J. Kocot ${ }^{152}$, K. Kohri ${ }^{182}$, K. Kokkotas ${ }^{69}$, N. Komin ${ }^{58}$, A. Kong ${ }^{2}$, K. Kosack ${ }^{4}$, G. Kowal ${ }^{132}$, F. Krack ${ }^{52}$, M. Krause ${ }^{52}$, F. Krennrich ${ }^{129}$, M. Krumholz ${ }^{70}$, H. Kubo ${ }^{180}$, V. Kudryavtsev ${ }^{183}$, S. Kunwar ${ }^{53}$, Y. Kuroda ${ }^{139}$, J. Kushida ${ }^{157}$, P. Kushwaha ${ }^{19}$, A. La Barbera ${ }^{91}$, N. La Palombara ${ }^{61}$, V. La Parola ${ }^{91}$, G. La Rosa ${ }^{91}$, R. Lahmann ${ }^{142}$, G. Lamanna ${ }^{45}$, A. Lamastra ${ }^{28}$, M. Landoni ${ }^{95}$, D. Landriu ${ }^{4}$, R.G. Lang ${ }^{80}$, J. Lapington ${ }^{124}$, P. Laporte ${ }^{20}$, P. Lason ${ }^{152}$, J. Lasuik ${ }^{37}$, J. Lazendic-Galloway ${ }^{7}$, T. Le Flour ${ }^{45}$, P. Le Sidaner ${ }^{20}$, S. Leach ${ }^{124}$, A. Leckngam ${ }^{31}$, S.-H. Lee ${ }^{180}$, W.H. Lee ${ }^{16}$, S. Lee ${ }^{118}$, M.A. Leigui de Oliveira $^{184}$, A. Lemière ${ }^{85}$, M. Lemoine-Goumard ${ }^{106}$, J.-P. Lenain ${ }^{79}$, F. Leone ${ }^{94,185}$, V. Leray ${ }^{8}$, G. Leto ${ }^{29}$, F. Leuschner ${ }^{69}$, C. Levy ${ }^{79,20}$, R. Lindemann ${ }^{52}$, E. Lindfors ${ }^{65}$, L. Linhoff ${ }^{46}$, I. Liodakis ${ }^{65}$, A. Lipniacka ${ }^{16}$, S. Lloyd ${ }^{5}$, M. Lobo ${ }^{113}$, T. Lohse ${ }^{186}$, S. Lombardi ${ }^{28}$, F. Longo ${ }^{145}$, A. Lopez $^{32}$, M. López ${ }^{11}$, R. López-Coto ${ }^{55}$, S. Loporchio ${ }^{149}$, F. Louis ${ }^{75}$, M. Louys ${ }^{20}$, F. Lucarelli ${ }^{28}$, D. Lucchesi ${ }^{55}$, H. Ludwig Boudi ${ }^{39}$, P.L. Luque-Escamilla ${ }^{56}$, E. Lyard $^{38}$, M.C. Maccarone ${ }^{91}$, T. Maccarone ${ }^{187}$, E. Mach ${ }^{101}$, A.J. Maciejewski ${ }^{188}$, J. Mackey ${ }^{15}$, G.M. Madejski ${ }^{96}$, P. Maeght ${ }^{39}$, C. Maggio ${ }^{138}$, G. Maier ${ }^{52}$, A. Majczyna ${ }^{126}$, P. Majumdar ${ }^{83,2}$, M. Makariev ${ }^{189}$, M. Mallamaci ${ }^{55}$, R. Malta Nunes de Almeida ${ }^{184}$, S. Maltezos ${ }^{134}$, D. Malyshev ${ }^{142}$, D. Malyshev ${ }^{69}$, D. Mandat ${ }^{33}$, G. Maneva ${ }^{189}$, M. Manganaro ${ }^{121}$, G. Manicò ${ }^{94}$, P. Manigot ${ }^{8}$, K. Mannheim ${ }^{122}$, N. Maragos ${ }^{134}$, D. Marano ${ }^{29}$, M. Marconi ${ }^{84}$, A. Marcowith ${ }^{39}$, M. Marculewicz ${ }^{190}$, B. Marčun ${ }^{68}$, J. Marín ${ }^{98}$, N. Marinello ${ }^{55}$, P. Marinos ${ }^{118}$, M. Mariotti ${ }^{55}$, S. Markoff ${ }^{174}$, P. Marquez ${ }^{41}$, G. Marsella ${ }^{94}$, J. Martít ${ }^{56}$ J.-M. Martin ${ }^{20}$, P. Martin ${ }^{87}$, O. Martinez ${ }^{30}$, M. Martínez ${ }^{41}$, G. Martínez ${ }^{113}$, O. Martínez ${ }^{41}$, H. Martínez-Huerta ${ }^{80}$ C. $\operatorname{Marty}^{87}$, R. Marx ${ }^{53}$, N. Masetti ${ }^{21,151}$, P. Massimino ${ }^{29}$, A. Mastichiadis ${ }^{191}$, H. Matsumoto ${ }^{167}$, N. Matthews ${ }^{164}$, G. Maurin ${ }^{45}$, W. Max-Moerbeck ${ }^{192}$, N. Maxted $^{43}$, D. Mazin²,105, M.N. Mazziotta ${ }^{120}$, S.M. Mazzola ${ }^{77}$, J.D. Mbarubucyeye ${ }^{52}$, L. Mc Comb ${ }^{5}$, I. McHardy ${ }^{115}$, S. McKeague ${ }^{107}$, S. McMuldroch ${ }^{63}$, E. Medina ${ }^{64}$, D. Medina Miranda ${ }^{17}$, A. Melandri ${ }^{95}$, C. Melioli ${ }^{19}$, D. Melkumyan ${ }^{52}$, S. Menchiari ${ }^{62}$, S. Mender ${ }^{46}$, S. Mereghetti ${ }^{61}$, G. Merino Arévalo ${ }^{6}$, E. Mestre ${ }^{13}$, J.-L. Meunier ${ }^{79}$, T. Meures ${ }^{135}$, M. Meyer ${ }^{142}$, S. Micanovic ${ }^{121}$, M. Miceli ${ }^{77}$, M. Michailidis ${ }^{69}$, J. Michałowski ${ }^{101}$, T. Miener ${ }^{11}$, I. Mievre ${ }^{45}$, J. Miller ${ }^{35}$, I.A. Minaya ${ }^{153}$, T. Mineo ${ }^{91}$, M. Minev ${ }^{189}$, J.M. Miranda ${ }^{30}$, R. Mirzoyan ${ }^{105}$, A. Mitchell ${ }^{36}$, T. Mizuno ${ }^{193}$, B. Mode ${ }^{135}$, R. Moderski ${ }^{49}$, L. Mohrmann ${ }^{142}$, E. Molina ${ }^{81}$, E. Molinari ${ }^{148}$, T. Montaruli ${ }^{17}$, I. Monteiro ${ }^{45}$, C. Moore ${ }^{124}$, A. Moralejo ${ }^{41}$, D. Morcuende-Parrilla ${ }^{11}$, E. Moretti ${ }^{41}$, L. Morganti ${ }^{64}$, K. Mori ${ }^{194}$, P. Moriarty ${ }^{15}$, K. Morik ${ }^{46}$, G. Morlino ${ }^{22}$, P. Morris ${ }^{14}$, A. Morselli ${ }^{25}$, K. Mosshammer ${ }^{52}$, P. Moya ${ }^{192}$, R. Mukherjee ${ }^{9}$, J. Muller ${ }^{8}$, C. Mundell ${ }^{172}$, J. Mundet ${ }^{41}$, T. Murach ${ }^{52}$, A. Muraczewski ${ }^{49}$, H. Muraishi $^{195}$, K. Murase ${ }^{2}$, I. Musella ${ }^{84}$, A. Musumarra ${ }^{120}$, A. Nagai ${ }^{17}$, N. Nagar ${ }^{196}$, S. Nagataki ${ }^{54}$, T. Naito ${ }^{156}$, T. Nakamori ${ }^{154}$, K. Nakashima ${ }^{142}$, K. Nakayama ${ }^{51}$, N. Nakhjiri ${ }^{13}$, G. Naletto ${ }^{55}$, D. Naumann ${ }^{52}$, L. Nava ${ }^{95}$, R. Navarro ${ }^{174}$, M.A. Nawaz ${ }^{132}$, H. Ndiyavala $^{1}$, D. Neise ${ }^{36}$, L. Nellen ${ }^{16}$, R. Nemmen ${ }^{19}$, M. Newbold ${ }^{164}$, N. Neyroud ${ }^{45}$, K. Ngernphat ${ }^{31}$, T. Nguyen Trung ${ }^{73}$, L. Nicastro ${ }^{21}$, L. Nickel ${ }^{46}$, J. Niemiec ${ }^{101}$, D. Nieto ${ }^{11}$, M. Nievas ${ }^{32}$, C. Nigro ${ }^{41}$,

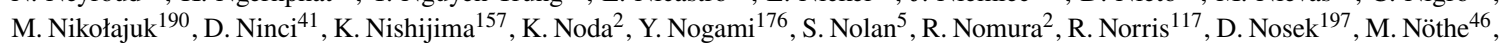
B. Novosyadlyj ${ }^{198}$, V. Novotny ${ }^{197}$, S. Nozaki ${ }^{180}$, F. Nunio ${ }^{144}$, P. O’Brien ${ }^{124}$, K. Obara ${ }^{176}$, R. Oger ${ }^{85}$, Y. Ohira ${ }^{51}$, M. Ohishi ${ }^{2}$, S. $\mathrm{Ohm}^{52}$, Y. Ohtani ${ }^{2}$, T. Oka ${ }^{180}$, N. Okazaki ${ }^{2}$, A. Okumura ${ }^{139,199}$, J.-F. Olive ${ }^{87}$, C. Oliver ${ }^{30}$, G. Olivera ${ }^{52}$, B. Olmi ${ }^{22}$, R.A. Ong ${ }^{71}$, M. Orienti ${ }^{90}$, R. Orito ${ }^{200}$, M. Orlandini ${ }^{21}$, S. Orlando ${ }^{77}$, E. Orlando ${ }^{145}$, J.P. Osborne ${ }^{124}$, M. Ostrowski ${ }^{169}$, N. Otte ${ }^{146}$, E. Ovcharov $^{86}$, E. Owen ${ }^{2}$, I. Oya ${ }^{159}$, A. Ozieblo ${ }^{152}$, M. Padovani ${ }^{22}$, I. Pagano ${ }^{29}$, A. Pagliaro ${ }^{91}$, A. Paizis ${ }^{61}$, M. Palatiello ${ }^{145}$, M. Palatka ${ }^{33}$, E. Palazzi ${ }^{21}$, J.-L. Panazol ${ }^{45}$, D. Paneque ${ }^{105}$, B. Panes ${ }^{3}$, S. Panny ${ }^{163}$, F.R. Pantaleo ${ }^{72}$, M. Panter ${ }^{53}$, R. Paoletti ${ }^{62}$, M. Paolillo ${ }^{24,110}$, A. Papitto $^{28}$, A. Paravac ${ }^{122}$, J.M. Paredes ${ }^{81}$, G. Pareschi ${ }^{95}$, N. Park ${ }^{127}$, N. Parmiggiani ${ }^{21}$, R.D. Parsons ${ }^{186}$, P. Paśko ${ }^{201}$, S. Patel ${ }^{52}$, B. Patricellii ${ }^{28}$, G. Pauletta ${ }^{103}$, L. Pavletić ${ }^{121}$, S. Pavy ${ }^{8}$, A. Pe'er ${ }^{105}$, M. Pech ${ }^{33}$, M. Pecimotika ${ }^{121}$, M.G. Pellegriti ${ }^{120}$, P. Peñil Del $\mathrm{Campo}^{11}$, M. Penno ${ }^{52}$, A. Pepato ${ }^{55}$, S. Perard ${ }^{106}$, C. Perennes ${ }^{55}$, G. Peres ${ }^{77}$, M. Peresano ${ }^{4}$, A. Pérez-Aguilera ${ }^{11}$, J. Pérez-Romero ${ }^{14}$, M.A. Pérez-Torres ${ }^{12}$, M. Perri ${ }^{28}$, M. Persic ${ }^{103}$, S. Petrera ${ }^{18}$, P.-O. Petrucci ${ }^{125}$, O. Petruk ${ }^{66}$, B. Peyaud ${ }^{89}$, K. Pfrang ${ }^{52}$, E. Pian ${ }^{21}$, G. Piano ${ }^{99}$, P. Piatteli ${ }^{44}$, E. Pietropaolo ${ }^{18}$, R. Pillera ${ }^{149}$, B. Pilszyk ${ }^{101}$, D. Pimentel ${ }^{202}$, F. Pintore ${ }^{91}$, C. Pio García ${ }^{41}$, G. Pirola $^{64}$, F. Piron ${ }^{39}$, A. Pisarski ${ }^{190}$, S. Pita ${ }^{85}$, M. Pohl ${ }^{128}$, V. Poireau ${ }^{45}$, P. Poledrelli ${ }^{159}$, A. Pollo ${ }^{126}$, M. Polo ${ }^{113}$, C. Pongkitivanichkul ${ }^{31}$,

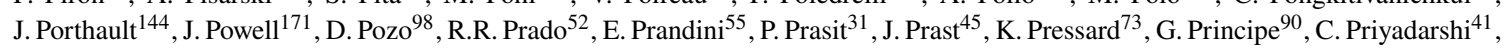


N. Produit ${ }^{38}$, D. Prokhorov ${ }^{174}$, H. Prokoph ${ }^{52}$, M. Prouza $^{33}$, H. Przybilski $^{101}$, E. Pueschel ${ }^{52}$, G. Pühlhofer ${ }^{69}$, I. Puljak ${ }^{150}$, M.L. Pumo ${ }^{94}$, M. Punch ${ }^{85,57}$, F. Queiroz ${ }^{203}$, J. Quinn ${ }^{204}$, A. Quirrenbach ${ }^{170}$, S. Rainò ${ }^{149}$, P.J. Rajda ${ }^{175}$, R. Rando ${ }^{55}$, S. Razzaque ${ }^{205}$, E. Rebert ${ }^{20}$, S. Recchia ${ }^{85}$, P. Reichherzer ${ }^{59}$, O. Reimer ${ }^{163}$, A. Reimer ${ }^{163}$, A. Reisenegger ${ }^{3,206}$, Q. Remy ${ }^{53}$, M. Renaud ${ }^{39}$, T. Reposeur ${ }^{106}$, B. Reville ${ }^{53}$, J.-M. Reymond ${ }^{75}$, J. Reynolds ${ }^{15}$, W. Rhode ${ }^{46}$, D. Ribeiro ${ }^{9}$, M. Ribó ${ }^{81}$, G. Richards ${ }^{162}$, T. Richtler ${ }^{196}$, J. Rico ${ }^{41}$, F. Rieger ${ }^{53}$, L. Riitano ${ }^{135}$, V. Ripepi ${ }^{84}$, M. Riquelme ${ }^{192}$, D. Riquelme ${ }^{35}$, S. Rivoire ${ }^{39}$, V. Rizi ${ }^{18}$, E. Roache ${ }^{63}$, B. Röben ${ }^{159}$, M. Roche ${ }^{106}$, J. Rodriguez ${ }^{4}$, G. Rodriguez Fernandez ${ }^{25}$, J.C. Rodriguez Ramirez ${ }^{19}$, J.J. Rodríguez Vázquez ${ }^{113}$, F. Roepke ${ }^{170}$, G. Rojas $^{207}$, L. Romanato ${ }^{55}$, P. Romano ${ }^{95}$, G. Romeo ${ }^{29}$, F. Romero Lobato ${ }^{11}$, C. Romoli ${ }^{53}$, M. Roncadelli ${ }^{103}$, S. Ronda ${ }^{30}$, J. Rosado ${ }^{11}$, A. Rosales de Leon $^{5}$, G. Rowell ${ }^{118}$, B. Rudak ${ }^{49}$, A. Rugliancich ${ }^{74}$, J.E. Ruíz del Mazo ${ }^{12}$, W. Rujopakarn ${ }^{31}$, C. Rulten ${ }^{5}$, C. Russell ${ }^{3}$, F. Russo ${ }^{21}$, I. Sadeh ${ }^{52}$, E. Sæther Hatlen ${ }^{10}$, S. Safi-Harb ${ }^{37}$, L. Saha ${ }^{11}$, P. Saha ${ }^{208}$, V. Sahakian ${ }^{147}$, S. Sailer ${ }^{53}$, T. Saito ${ }^{2}$, N. Sakaki ${ }^{54}$, S. Sakurai ${ }^{2}$, F. Salesa Greus ${ }^{101}$, G. Salina ${ }^{25}$, H. Salzmann ${ }^{69}$, D. Sanchez ${ }^{45}$, M. Sánchez-Conde ${ }^{14}$, H. Sandaker ${ }^{10}$, A. Sandoval ${ }^{16}$, P. Sangiorgi ${ }^{91}$, M. Sanguillon ${ }^{39}$, H. Sano ${ }^{2}$, M. Santander ${ }^{171}$, A. Santangelo ${ }^{69}$, E.M. Santos ${ }^{202}$, R. Santos-Lima ${ }^{19}$, A. Sanuy ${ }^{81}$, L. Sapozhnikov ${ }^{96}$, T. Saric ${ }^{150}$, S. Sarkar ${ }^{14}$, H. Sasaki ${ }^{157}$, N. Sasaki ${ }^{179}$, K. Satalecka ${ }^{52}$, Y. Sato ${ }^{209}$, F.G. Saturni ${ }^{28}$, M. Sawada ${ }^{54}$, U. Sawangwit ${ }^{31}$, J. Schaefer ${ }^{142}$, A. Scherer ${ }^{3}$, J. Scherpenberg ${ }^{105}$, P. Schipani ${ }^{44}$, B. Schleicher ${ }^{122}$, J. Schmoll ${ }^{5}$, M. Schneider ${ }^{143}$, H. Schoorlemmer ${ }^{53}$, P. Schovanek ${ }^{33}$, F. Schussler ${ }^{89}$, B. Schwab ${ }^{142}$, U. Schwanke ${ }^{186}$, J. Schwarz ${ }^{95}$, T. Schweizer ${ }^{105}$, E. Sciacca ${ }^{29}$, S. Scuderi ${ }^{61}$, M. Seglar Arroyo ${ }^{45}$, A. Segreto ${ }^{91}$, I. Seitenzahl ${ }^{43}$, D. Semikoz ${ }^{85}$, O. Sergijenko ${ }^{136}$, J.E. Serna Franco ${ }^{16}$, M. Servillat ${ }^{20}$, K. Seweryn ${ }^{201}$, V. Sguera ${ }^{21}$, A. Shalchi ${ }^{37}$, R.Y. Shang ${ }^{71}$, P. Sharma ${ }^{73}$, R.C. Shellard ${ }^{40}$, L. Sidoli ${ }^{61}$, J. Sieiro ${ }^{81}$, H. Siejkowski ${ }^{152}$, J. Silk ${ }^{114}$, A. Sillanpää ${ }^{65}$, B.B. Singh ${ }^{109}$, K.K. Singh ${ }^{210}$, A. Sinha ${ }^{39}$, C. Siqueira ${ }^{80}$, G. Sironi ${ }^{95}$, J. Sitarek ${ }^{60}$, P. Sizun ${ }^{75}$, V. Sliusar ${ }^{38}$, A. Slowikowska ${ }^{178}$, D. Sobczyńska ${ }^{60}$, R.W. Sobrinho ${ }^{184}$, H. Sol $^{20}$, G. Sottile ${ }^{91}$, H. Spackman ${ }^{114}$, A. Specovius ${ }^{142}$, S. Spencer ${ }^{114}$, G. Spengler ${ }^{186}$, D. Spiga ${ }^{95}$, A. Spolon ${ }^{55}$, W. Springer ${ }^{164}$, A. Stamerra ${ }^{28}$, S. Stanič ${ }^{68}$, R. Starling ${ }^{124}$, L. Stawarz ${ }^{169}$, R. Steenkamp ${ }^{48}$, S. Stefanik ${ }^{197}$, C. Stegmann ${ }^{128}$, A. Steiner ${ }^{52}$, S. Steinmass ${ }^{53}$, C. Stella ${ }^{103}$, C. Steppa ${ }^{128}$, R. Sternberger ${ }^{52}$, M. Sterzel ${ }^{152}$, C. Stevens ${ }^{135}$, B. Stevenson ${ }^{71}$, T. Stolarczyk ${ }^{4}$, G. Stratta ${ }^{21}$, U. Straumann ${ }^{208}$, J. Strišković ${ }^{166}$, M. Strzys ${ }^{2}$, R. Stuik ${ }^{174}$, M. Suchenek ${ }^{211}$, Y. Suda ${ }^{140}$, Y. Sunada $^{179}$, T. Suomijarvi ${ }^{73}$, T. Suric ${ }^{212}$, P. Sutcliffe ${ }^{153}$, H. Suzuki $^{213}$, P. Swierk ${ }^{101}$, T. Szepieniec ${ }^{152}$, A. Tacchini ${ }^{21}$, K. Tachihara ${ }^{141}$, G. Tagliaferrii ${ }^{95}$, H. Tajima ${ }^{139}$, N. Tajima ${ }^{2}$, D. Tak ${ }^{52}$, K. Takahashi ${ }^{214}$, H. Takahashi ${ }^{140}$, M. Takahashi ${ }^{2}$, M. Takahashi ${ }^{2}$, J. Takata $^{2}$, R. Takeishi ${ }^{2}$, T. Tam $^{2}$, M. Tanaka ${ }^{182}$, T. Tanaka ${ }^{213}$, S. Tanaka ${ }^{209}$, D. Tateishi ${ }^{179}$, M. Tavani ${ }^{99}$, F. Tavecchio ${ }^{95}$, T. Tavernier $^{89}$, L. Taylor ${ }^{135}$, A. Taylor ${ }^{52}$, L.A. Tejedor ${ }^{11}$, P. Temnikov ${ }^{189}$, Y. Terada ${ }^{179}$, K. Terauchi ${ }^{180}$, J.C. Terrazas ${ }^{192}$, R. Terrier ${ }^{85}$, T. Terzic ${ }^{121}$, M. Teshima ${ }^{105,2}$, V. Testa ${ }^{28}$, D. Thibaut ${ }^{85}$, F. Thocquenne ${ }^{75}$, W. Tian ${ }^{2}$, L. Tibaldo ${ }^{87}$, A. Tiengo ${ }^{215}$, D. Tiziani ${ }^{142}$, M. Tluczykont $^{50}$, C.J. Todero Peixoto ${ }^{102}$, F. Tokanai ${ }^{154}$, K. Toma ${ }^{160}$, L. Tomankova ${ }^{142}$, J. Tomastik ${ }^{104}$, D. Tonev ${ }^{189}$, M. Tornikoski ${ }^{216}$, D.F. Torres ${ }^{13}$, E. Torresi ${ }^{21}$, G. Tosti ${ }^{95}$, L. Tosti ${ }^{23}$, T. Totani ${ }^{51}$, N. Tothill ${ }^{117}$, F. Toussenel ${ }^{79}$, G. Tovmassian ${ }^{16}$, P. Travnicek ${ }^{33}$, C. Trichard $^{8}$,

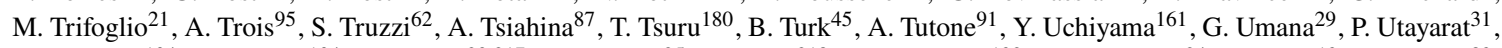
L. Vaclavek ${ }^{104}$, M. Vacula ${ }^{104}$, V. Vagelli ${ }^{23,217}$, F. Vagnetti ${ }^{25}$, F. Vakili ${ }^{218}$, J.A. Valdivia ${ }^{192}$, M. Valentino ${ }^{24}$, A. Valio ${ }^{19}$, B. Vallage ${ }^{89}$, P. Vallania ${ }^{44,64}$, J.V. Valverde Quispe ${ }^{8}$, A.M. Van den Berg ${ }^{42}$, W. van Driel ${ }^{20}$, C. van Eldik ${ }^{142}$, C. van Rensburg ${ }^{1}$, B. van Soelen ${ }^{210}$, J. Vandenbroucke ${ }^{135}$, J. Vanderwalt ${ }^{1}$, G. Vasileiadis ${ }^{39}$, V. Vassiliev ${ }^{71}$, M. Vázquez Acosta ${ }^{32}$, M. Vecchi ${ }^{42}$, A. Vega ${ }^{98}$, J. Veh $^{142}$, P. Veitch ${ }^{118}$, P. Venault ${ }^{75}$, C. Venter ${ }^{1}$, S. Ventura ${ }^{62}$, S. Vercellone ${ }^{95}$, S. Vergani ${ }^{20}$, V. Verguilov ${ }^{189}$, G. Verna ${ }^{27}$, S. Vernetto ${ }^{44,64}$, V. Verzi ${ }^{25}$, G.P. Vettolani ${ }^{90}$, C. Veyssiere ${ }^{144}$, I. Viale ${ }^{55}$, A. Viana ${ }^{80}$, N. Viaux ${ }^{35}$, J. Vicha ${ }^{33}$, J. Vignatti ${ }^{35}$, C.F. Vigorito ${ }^{64,108}$, J. Villanueva ${ }^{98}$, J. Vink ${ }^{174}$, V. Vitale ${ }^{23}$, V. Vittorini ${ }^{99}$, V. Vodeb ${ }^{68}$, H. Voelk ${ }^{53}$, N. Vogel ${ }^{142}$, V. Voisin ${ }^{79}$, S. Vorobiov ${ }^{68}$, I. Vovk ${ }^{2}$, M. Vrastil ${ }^{33}$, T. Vuillaume ${ }^{45}$, S.J. Wagner ${ }^{170}$, R. Wagner ${ }^{105}$, P. Wagner ${ }^{52}$, K. Wakazono ${ }^{139}$, S.P. Wakely ${ }^{127}$, R. Walter ${ }^{38}$, M. Ward ${ }^{5}$,

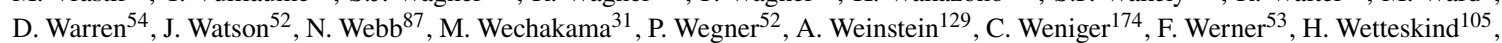
M. White ${ }^{118}$, R. White ${ }^{53}$, A. Wierzcholska ${ }^{101}$, S. Wiesand ${ }^{52}$, R. Wijers ${ }^{174}$, M. Wilkinson ${ }^{124}$, M. Will ${ }^{105}$, D.A. Williams ${ }^{143}$, J. Williams ${ }^{124}$, T. Williamson ${ }^{162}$, A. Wolter ${ }^{95}$, Y.W. Wong ${ }^{142}$, M. Wood ${ }^{96}$, C. Wunderlich ${ }^{62}$, T. Yamamoto ${ }^{213}$, H. Yamamoto $^{141}$, Y. Yamane ${ }^{141}$, R. Yamazaki ${ }^{209}$, S. Yanagita ${ }^{176}$, L. Yang ${ }^{205}$, S. Yoo ${ }^{180}$, T. Yoshida ${ }^{176}$, T. Yoshikoshi ${ }^{2}$, P. Yu ${ }^{71}$, P. Yu ${ }^{85}$, A. Yusafzai ${ }^{59}$, M. Zacharias ${ }^{20}$, G. Zaharijas ${ }^{68}$, B. Zaldivar ${ }^{14}$, L. Zampieri ${ }^{76}$, R. Zanmar Sanchez ${ }^{29}$, D. Zaric ${ }^{150}$, M. Zavrtanik ${ }^{68}$, D. Zavrtanik ${ }^{68}$, A.A. Zdziarski ${ }^{49}$, A. Zech ${ }^{20}$, H. Zechlin ${ }^{64}$, A. Zenin ${ }^{139}$, A. Zerwekh ${ }^{35}$, V.I. Zhdanov ${ }^{136}$, K. Ziętara ${ }^{169}$, A. Zink ${ }^{142}$, J. Ziółkowski ${ }^{49}$, V. Zitelli ${ }^{21}$, M. Živec ${ }^{68}$, A. Zmija ${ }^{142}$

1. Centre for Space Research, North-West University, Potchefstroom, 2520, South Africa

2. Institute for Cosmic Ray Research, University of Tokyo, 5-1-5, Kashiwa-no-ha, Kashiwa, Chiba 277-8582, Japan

3. Pontificia Universidad Católica de Chile, Av. Libertador Bernardo O'Higgins 340, Santiago, Chile

4. AIM, CEA, CNRS, Université Paris-Saclay, Université Paris Diderot, Sorbonne Paris Cité, CEA Paris-Saclay, IRFU/DAp, Bat 709, Orme des Merisiers, 91191 Gif-sur-Yvette, France

5. Centre for Advanced Instrumentation, Dept. of Physics, Durham University, South Road, Durham DH1 3LE, United Kingdom

6. Port d'Informació Científica, Edifici D, Carrer de l'Albareda, 08193 Bellaterrra (Cerdanyola del Vallès), Spain

7. School of Physics and Astronomy, Monash University, Melbourne, Victoria 3800, Australia

8. Laboratoire Leprince-Ringuet, École Polytechnique (UMR 7638, CNRS/IN2P3, Institut Polytechnique de Paris), 91128 Palaiseau, France

9. Department of Physics, Columbia University, 538 West 120th Street, New York, NY 10027, USA

10. University of Oslo, Department of Physics, Sem Saelandsvei 24 - PO Box 1048 Blindern, N-0316 Oslo, Norway

11. EMFTEL department and IPARCOS, Universidad Complutense de Madrid, 28040 Madrid, Spain

12. Instituto de Astrofísica de Andalucía-CSIC, Glorieta de la Astronomía s/n, 18008, Granada, Spain

13. Institute of Space Sciences (ICE-CSIC), and Institut d'Estudis Espacials de Catalunya (IEEC), and Institució Catalana de Recerca I Estudis Avançats (ICREA), Campus UAB, Carrer de Can Magrans, s/n 08193 Cerdanyola del Vallés, Spain

14. Instituto de Física Teórica UAM/CSIC and Departamento de Física Teórica, Universidad Autónoma de Madrid, c/ Nicolás Cabrera 13-15, Campus de Cantoblanco UAM, 28049 Madrid, Spain 
15. Dublin Institute for Advanced Studies, 31 Fitzwilliam Place, Dublin 2, Ireland

16. Universidad Nacional Autónoma de México, Delegación Coyoacán, 04510 Ciudad de México, Mexico

17. University of Geneva - Département de physique nucléaire et corpusculaire, 24 rue du Général-Dufour, 1211 Genève 4, Switzerland

18. INFN Dipartimento di Scienze Fisiche e Chimiche - Università degli Studi dell'Aquila and Gran Sasso Science Institute, Via Vetoio 1, Viale Crispi 7, 67100 L'Aquila, Italy

19. Instituto de Astronomia, Geofísico, e Ciências Atmosféricas - Universidade de São Paulo, Cidade Universitária, R. do Matão, 1226, CEP 05508-090, São Paulo, SP, Brazil

20. LUTH, GEPI and LERMA, Observatoire de Paris, CNRS, PSL University, 5 place Jules Janssen, 92190, Meudon, France

21. INAF - Osservatorio di Astrofisica e Scienza dello spazio di Bologna, Via Piero Gobetti 93/3, 40129 Bologna, Italy

22. INAF - Osservatorio Astrofisico di Arcetri, Largo E. Fermi, 5 - 50125 Firenze, Italy

23. INFN Sezione di Perugia and Università degli Studi di Perugia, Via A. Pascoli, 06123 Perugia, Italy

24. INFN Sezione di Napoli, Via Cintia, ed. G, 80126 Napoli, Italy

25. INFN Sezione di Roma Tor Vergata, Via della Ricerca Scientifica 1, 00133 Rome, Italy

26. Argonne National Laboratory, 9700 S. Cass Avenue, Argonne, IL 60439, USA

27. Aix-Marseille Université, CNRS/IN2P3, CPPM, 163 Avenue de Luminy, 13288 Marseille cedex 09, France

28. INAF - Osservatorio Astronomico di Roma, Via di Frascati 33, 00040, Monteporzio Catone, Italy

29. INAF - Osservatorio Astrofisico di Catania, Via S. Sofia, 78, 95123 Catania, Italy

30. Grupo de Electronica, Universidad Complutense de Madrid, Av. Complutense s/n, 28040 Madrid, Spain

31. National Astronomical Research Institute of Thailand, 191 Huay Kaew Rd., Suthep, Muang, Chiang Mai, 50200, Thailand

32. Instituto de Astrofísica de Canarias and Departamento de Astrofísica, Universidad de La Laguna, La Laguna, Tenerife, Spain

33. FZU - Institute of Physics of the Czech Academy of Sciences, Na Slovance 1999/2, 18221 Praha 8, Czech Republic

34. Astronomical Institute of the Czech Academy of Sciences, Bocni II 1401 - 14100 Prague, Czech Republic

35. CCTVal, Universidad Técnica Federico Santa María, Avenida España 1680, Valparaíso, Chile

36. ETH Zurich, Institute for Particle Physics, Schafmattstr. 20, CH-8093 Zurich, Switzerland

37. The University of Manitoba, Dept of Physics and Astronomy, Winnipeg, Manitoba R3T 2N2, Canada

38. Department of Astronomy, University of Geneva, Chemin d'Ecogia 16, CH-1290 Versoix, Switzerland

39. Laboratoire Univers et Particules de Montpellier, Université de Montpellier, CNRS/IN2P3, CC 72, Place Eugène Bataillon, F-34095 Montpellier Cedex 5, France

40. Centro Brasileiro de Pesquisas Físicas, Rua Xavier Sigaud 150, RJ 22290-180, Rio de Janeiro, Brazil

41. Institut de Fisica d'Altes Energies (IFAE), The Barcelona Institute of Science and Technology, Campus UAB, 08193 Bellaterra (Barcelona), Spain

42. University of Groningen, KVI - Center for Advanced Radiation Technology, Zernikelaan 25, 9747 AA Groningen, The Netherlands

43. School of Physics, University of New South Wales, Sydney NSW 2052, Australia

44. INAF - Osservatorio Astrofisico di Torino, Strada Osservatorio 20, 10025 Pino Torinese (TO), Italy

45. Univ. Savoie Mont Blanc, CNRS, Laboratoire d'Annecy de Physique des Particules - IN2P3, 74000 Annecy, France

46. Department of Physics, TU Dortmund University, Otto-Hahn-Str. 4, 44221 Dortmund, Germany

47. University of Zagreb, Faculty of electrical engineering and computing, Unska 3, 10000 Zagreb, Croatia

48. University of Namibia, Department of Physics, 340 Mandume Ndemufayo Ave., Pioneerspark, Windhoek, Namibia

49. Nicolaus Copernicus Astronomical Center, Polish Academy of Sciences, ul. Bartycka 18, 00-716 Warsaw, Poland

50. Universität Hamburg, Institut für Experimentalphysik, Luruper Chaussee 149, 22761 Hamburg, Germany

51. Graduate School of Science, University of Tokyo, 7-3-1 Hongo, Bunkyo-ku, Tokyo 113-0033, Japan

52. Deutsches Elektronen-Synchrotron, Platanenallee 6, 15738 Zeuthen, Germany

53. Max-Planck-Institut für Kernphysik, Saupfercheckweg 1, 69117 Heidelberg, Germany

54. RIKEN, Institute of Physical and Chemical Research, 2-1 Hirosawa, Wako, Saitama, 351-0198, Japan

55. INFN Sezione di Padova and Università degli Studi di Padova, Via Marzolo 8, 35131 Padova, Italy

56. Escuela Politécnica Superior de Jaén, Universidad de Jaén, Campus Las Lagunillas s/n, Edif. A3, 23071 Jaén, Spain

57. Department of Physics and Electrical Engineering, Linnaeus University, 35195 Växjö, Sweden

58. University of the Witwatersrand, 1 Jan Smuts Avenue, Braamfontein, 2000 Johannesburg, South Africa

59. Institut für Theoretische Physik, Lehrstuhl IV: Plasma-Astroteilchenphysik, Ruhr-Universität Bochum, Universitätsstraße 150, 44801 Bochum, Germany

60. Faculty of Physics and Applied Computer Science, University of Lódź, ul. Pomorska 149-153, 90-236 Lódź, Poland

61. INAF - Istituto di Astrofisica Spaziale e Fisica Cosmica di Milano, Via A. Corti 12, 20133 Milano, Italy

62. INFN and Università degli Studi di Siena, Dipartimento di Scienze Fisiche, della Terra e dell'Ambiente (DSFTA), Sezione di Fisica, Via Roma 56, 53100 Siena, Italy

63. Center for Astrophysics | Harvard \& Smithsonian, 60 Garden St, Cambridge, MA 02180, USA

64. INFN Sezione di Torino, Via P. Giuria 1, 10125 Torino, Italy

65. Finnish Centre for Astronomy with ESO, University of Turku, Finland, FI-20014 University of Turku, Finland

66. Pidstryhach Institute for Applied Problems in Mechanics and Mathematics NASU, 3B Naukova Street, Lviv, 79060, Ukraine

67. Bhabha Atomic Research Centre, Trombay, Mumbai 400085, India 
68. Center for Astrophysics and Cosmology, University of Nova Gorica, Vipavska 11c, 5270 Ajdovščina, Slovenia

69. Institut für Astronomie und Astrophysik, Universität Tübingen, Sand 1, 72076 Tübingen, Germany

70. Research School of Astronomy and Astrophysics, Australian National University, Canberra ACT 0200, Australia

71. Department of Physics and Astronomy, University of California, Los Angeles, CA 90095, USA

72. INFN Sezione di Bari and Politecnico di Bari, via Orabona 4, 70124 Bari, Italy

73. Laboratoire de Physique des 2 infinis, Irene Joliot-Curie,IN2P3/CNRS, Université Paris-Saclay, Université de Paris, 15 rue Georges Clemenceau, 91406 Orsay, Cedex, France

74. INFN Sezione di Pisa, Largo Pontecorvo 3, 56217 Pisa, Italy

75. IRFU/DEDIP, CEA, Université Paris-Saclay, Bat 141, 91191 Gif-sur-Yvette, France

76. INAF - Osservatorio Astronomico di Padova, Vicolo dell'Osservatorio 5, 35122 Padova, Italy

77. INAF - Osservatorio Astronomico di Palermo "G.S. Vaiana", Piazza del Parlamento 1, 90134 Palermo, Italy

78. School of Physics, University of Sydney, Sydney NSW 2006, Australia

79. Sorbonne Université, Université Paris Diderot, Sorbonne Paris Cité, CNRS/IN2P3, Laboratoire de Physique Nucléaire et de Hautes Energies, LPNHE, 4 Place Jussieu, F-75005 Paris, France

80. Instituto de Física de São Carlos, Universidade de São Paulo, Av. Trabalhador São-carlense, 400 - CEP 13566-590, São Carlos, SP, Brazil

81. Departament de Física Quàntica i Astrofísica, Institut de Ciències del Cosmos, Universitat de Barcelona, IEEC-UB, Martí i Franquès, 1, 08028, Barcelona, Spain

82. Department of Physics, Washington University, St. Louis, MO 63130, USA

83. Saha Institute of Nuclear Physics, Bidhannagar, Kolkata-700 064, India

84. INAF - Osservatorio Astronomico di Capodimonte, Via Salita Moiariello 16, 80131 Napoli, Italy

85. Université de Paris, CNRS, Astroparticule et Cosmologie, 10, rue Alice Domon et Léonie Duquet, 75013 Paris Cedex 13, France

86. Astronomy Department of Faculty of Physics, Sofia University, 5 James Bourchier Str., 1164 Sofia, Bulgaria

87. Institut de Recherche en Astrophysique et Planétologie, CNRS-INSU, Université Paul Sabatier, 9 avenue Colonel Roche, BP 44346, 31028 Toulouse Cedex 4, France

88. School of Physics and Astronomy, University of Minnesota, 116 Church Street S.E. Minneapolis, Minnesota 55455-0112, USA

89. IRFU, CEA, Université Paris-Saclay, Bât 141, 91191 Gif-sur-Yvette, France

90. INAF - Istituto di Radioastronomia, Via Gobetti 101, 40129 Bologna, Italy

91. INAF - Istituto di Astrofisica Spaziale e Fisica Cosmica di Palermo, Via U. La Malfa 153, 90146 Palermo, Italy

92. Astronomical Observatory, Department of Physics, University of Warsaw, Aleje Ujazdowskie 4, 00478 Warsaw, Poland

93. Armagh Observatory and Planetarium, College Hill, Armagh BT61 9DG, United Kingdom

94. INFN Sezione di Catania, Via S. Sofia 64, 95123 Catania, Italy

95. INAF - Osservatorio Astronomico di Brera, Via Brera 28, 20121 Milano, Italy

96. Kavli Institute for Particle Astrophysics and Cosmology, Department of Physics and SLAC National Accelerator Laboratory, Stanford University, 2575 Sand Hill Road, Menlo Park, CA 94025, USA

97. Universidade Cruzeiro do Sul, Núcleo de Astrofísica Teórica (NAT/UCS), Rua Galvão Bueno 8687, Bloco B, sala 16, Libertade 01506-000 - São Paulo, Brazil

98. Universidad de Valparaíso, Blanco 951, Valparaiso, Chile

99. INAF - Istituto di Astrofisica e Planetologia Spaziali (IAPS), Via del Fosso del Cavaliere 100, 00133 Roma, Italy

100. Lund Observatory, Lund University, Box 43, SE-22100 Lund, Sweden

101. The Henryk Niewodniczański Institute of Nuclear Physics, Polish Academy of Sciences, ul. Radzikowskiego 152, 31-342 Cracow, Poland

102. Escola de Engenharia de Lorena, Universidade de São Paulo, Área I - Estrada Municipal do Campinho, s/nº, CEP 12602-810, Pte. Nova, Lorena, Brazil

103. INFN Sezione di Trieste and Università degli Studi di Udine, Via delle Scienze 208, 33100 Udine, Italy

104. Palacky University Olomouc, Faculty of Science, RCPTM, 17. listopadu 1192/12, 77146 Olomouc, Czech Republic

105. Max-Planck-Institut für Physik, Föhringer Ring 6, 80805 München, Germany

106. CENBG, Univ. Bordeaux, CNRS-IN2P3, UMR 5797, 19 Chemin du Solarium, CS 10120, F-33175 Gradignan Cedex, France

107. Dublin City University, Glasnevin, Dublin 9, Ireland

108. Dipartimento di Fisica - Universitá degli Studi di Torino, Via Pietro Giuria 1 - 10125 Torino, Italy

109. Tata Institute of Fundamental Research, Homi Bhabha Road, Colaba, Mumbai 400005, India

110. Universitá degli Studi di Napoli "Federico II" - Dipartimento di Fisica "E. Pancini", Complesso universitario di Monte Sant'Angelo, Via Cintia - 80126 Napoli, Italy

111. Oskar Klein Centre, Department of Physics, University of Stockholm, Albanova, SE-10691, Sweden

112. Yale University, Department of Physics and Astronomy, 260 Whitney Avenue, New Haven, CT 06520-8101, USA

113. CIEMAT, Avda. Complutense 40, 28040 Madrid, Spain

114. University of Oxford, Department of Physics, Denys Wilkinson Building, Keble Road, Oxford OX1 3RH, United Kingdom

115. School of Physics \& Astronomy, University of Southampton, University Road, Southampton SO17 1BJ, United Kingdom

116. Department of Physics and Technology, University of Bergen, Museplass 1, 5007 Bergen, Norway

117. Western Sydney University, Locked Bag 1797, Penrith, NSW 2751, Australia 
118. School of Physical Sciences, University of Adelaide, Adelaide SA 5005, Australia

119. INFN Sezione di Roma La Sapienza, P.le Aldo Moro, 2 - 00185 Roma, Italy

120. INFN Sezione di Bari, via Orabona 4, 70126 Bari, Italy

121. University of Rijeka, Department of Physics, Radmile Matejcic 2, 51000 Rijeka, Croatia

122. Institute for Theoretical Physics and Astrophysics, Universität Würzburg, Campus Hubland Nord, Emil-Fischer-Str. 31, 97074 Würzburg, Germany

123. Universidade Federal Do Paraná - Setor Palotina, Departamento de Engenharias e Exatas, Rua Pioneiro, 2153, Jardim Dallas, CEP: 85950-000 Palotina, Paraná, Brazil

124. Dept. of Physics and Astronomy, University of Leicester, Leicester, LE1 7RH, United Kingdom

125. Univ. Grenoble Alpes, CNRS, IPAG, 414 rue de la Piscine, Domaine Universitaire, 38041 Grenoble Cedex 9, France

126. National Centre for nuclear research (Narodowe Centrum Badań Jądrowych), Ul. Andrzeja Sołtana7, 05-400 Otwock, Świerk, Poland

127. Enrico Fermi Institute, University of Chicago, 5640 South Ellis Avenue, Chicago, IL 60637, USA

128. Institut für Physik \& Astronomie, Universität Potsdam, Karl-Liebknecht-Strasse 24/25, 14476 Potsdam, Germany

129. Department of Physics and Astronomy, Iowa State University, Zaffarano Hall, Ames, IA 50011-3160, USA

130. School of Physics, Aristotle University, Thessaloniki, 54124 Thessaloniki, Greece

131. King's College London, Strand, London, WC2R 2LS, United Kingdom

132. Escola de Artes, Ciências e Humanidades, Universidade de São Paulo, Rua Arlindo Bettio, CEP 03828-000, 1000 São Paulo, Brazil

133. Dept. of Astronomy \& Astrophysics, Pennsylvania State University, University Park, PA 16802, USA

134. National Technical University of Athens, Department of Physics, Zografos 9, 15780 Athens, Greece

135. University of Wisconsin, Madison, 500 Lincoln Drive, Madison, WI, 53706, USA

136. Astronomical Observatory of Taras Shevchenko National University of Kyiv, 3 Observatorna Street, Kyiv, 04053, Ukraine

137. Department of Physics, Purdue University, West Lafayette, IN 47907, USA

138. Unitat de Física de les Radiacions, Departament de Física, and CERES-IEEC, Universitat Autònoma de Barcelona, Edifici C3, Campus UAB, 08193 Bellaterra, Spain

139. Institute for Space-Earth Environmental Research, Nagoya University, Chikusa-ku, Nagoya 464-8601, Japan

140. Department of Physical Science, Hiroshima University, Higashi-Hiroshima, Hiroshima 739-8526, Japan

141. Department of Physics, Nagoya University, Chikusa-ku, Nagoya, 464-8602, Japan

142. Friedrich-Alexander-Universität Erlangen-Nürnberg, Erlangen Centre for Astroparticle Physics (ECAP), Erwin-Rommel-Str. 1, 91058 Erlangen, Germany

143. Santa Cruz Institute for Particle Physics and Department of Physics, University of California, Santa Cruz, 1156 High Street, Santa Cruz, CA 95064, USA

144. IRFU / DIS, CEA, Université de Paris-Saclay, Bat 123, 91191 Gif-sur-Yvette, France

145. INFN Sezione di Trieste and Università degli Studi di Trieste, Via Valerio 2 I, 34127 Trieste, Italy

146. School of Physics \& Center for Relativistic Astrophysics, Georgia Institute of Technology, 837 State Street, Atlanta, Georgia, 30332-0430, USA

147. Alikhanyan National Science Laboratory, Yerevan Physics Institute, 2 Alikhanyan Brothers St., 0036, Yerevan, Armenia

148. INAF - Telescopio Nazionale Galileo, Roche de los Muchachos Astronomical Observatory, 38787 Garafia, TF, Italy

149. INFN Sezione di Bari and Università degli Studi di Bari, via Orabona 4, 70124 Bari, Italy

150. University of Split - FESB, R. Boskovica 32, 21000 Split, Croatia

151. Universidad Andres Bello, República 252, Santiago, Chile

152. Academic Computer Centre CYFRONET AGH, ul. Nawojki 11, 30-950 Cracow, Poland

153. University of Liverpool, Oliver Lodge Laboratory, Liverpool L69 7ZE, United Kingdom

154. Department of Physics, Yamagata University, Yamagata, Yamagata 990-8560, Japan

155. Astronomy Department, Adler Planetarium and Astronomy Museum, Chicago, IL 60605, USA

156. Faculty of Management Information, Yamanashi-Gakuin University, Kofu, Yamanashi 400-8575, Japan

157. Department of Physics, Tokai University, 4-1-1, Kita-Kaname, Hiratsuka, Kanagawa 259-1292, Japan

158. Centre for Astrophysics Research, Science \& Technology Research Institute, University of Hertfordshire, College Lane, Hertfordshire AL10 9AB, United Kingdom

159. Cherenkov Telescope Array Observatory, Saupfercheckweg 1, 69117 Heidelberg, Germany

160. Tohoku University, Astronomical Institute, Aobaku, Sendai 980-8578, Japan

161. Department of Physics, Rikkyo University, 3-34-1 Nishi-Ikebukuro, Toshima-ku, Tokyo, Japan

162. Department of Physics and Astronomy and the Bartol Research Institute, University of Delaware, Newark, DE 19716, USA

163. Institut für Astro- und Teilchenphysik, Leopold-Franzens-Universität, Technikerstr. 25/8, 6020 Innsbruck, Austria

164. Department of Physics and Astronomy, University of Utah, Salt Lake City, UT 84112-0830, USA

165. IMAPP, Radboud University Nijmegen, P.O. Box 9010, 6500 GL Nijmegen, The Netherlands

166. Josip Juraj Strossmayer University of Osijek, Trg Ljudevita Gaja 6, 31000 Osijek, Croatia

167. Department of Earth and Space Science, Graduate School of Science, Osaka University, Toyonaka 560-0043, Japan

168. Yukawa Institute for Theoretical Physics, Kyoto University, Kyoto 606-8502, Japan

169. Astronomical Observatory, Jagiellonian University, ul. Orla 171, 30-244 Cracow, Poland 
170. Landessternwarte, Zentrum für Astronomie der Universität Heidelberg, Königstuhl 12, 69117 Heidelberg, Germany

171. University of Alabama, Tuscaloosa, Department of Physics and Astronomy, Gallalee Hall, Box 870324 Tuscaloosa, AL 35487-0324, USA

172. Department of Physics, University of Bath, Claverton Down, Bath BA2 7AY, United Kingdom

173. University of Iowa, Department of Physics and Astronomy, Van Allen Hall, Iowa City, IA 52242, USA

174. Anton Pannekoek Institute/GRAPPA, University of Amsterdam, Science Park 9041098 XH Amsterdam, The Netherlands

175. Faculty of Computer Science, Electronics and Telecommunications, AGH University of Science and Technology, Kraków, al. Mickiewicza 30, 30-059 Cracow, Poland

176. Faculty of Science, Ibaraki University, Mito, Ibaraki, 310-8512, Japan

177. Faculty of Science and Engineering, Waseda University, Shinjuku, Tokyo 169-8555, Japan

178. Institute of Astronomy, Faculty of Physics, Astronomy and Informatics, Nicolaus Copernicus University in Toruń, ul. Grudziądzka 5, 87-100 Toruń, Poland

179. Graduate School of Science and Engineering, Saitama University, 255 Simo-Ohkubo, Sakura-ku, Saitama city, Saitama 338-8570, Japan

180. Division of Physics and Astronomy, Graduate School of Science, Kyoto University, Sakyo-ku, Kyoto, 606-8502, Japan

181. Centre for Quantum Technologies, National University Singapore, Block S15, 3 Science Drive 2, Singapore 117543, Singapore

182. Institute of Particle and Nuclear Studies, KEK (High Energy Accelerator Research Organization), 1-1 Oho, Tsukuba, 305-0801, Japan

183. Department of Physics and Astronomy, University of Sheffield, Hounsfield Road, Sheffield S3 7RH, United Kingdom

184. Centro de Ciências Naturais e Humanas, Universidade Federal do ABC, Av. dos Estados, 5001, CEP: 09.210-580, Santo André - SP, Brazil

185. Dipartimento di Fisica e Astronomia, Sezione Astrofisica, Universitá di Catania, Via S. Sofia 78, I-95123 Catania, Italy

186. Department of Physics, Humboldt University Berlin, Newtonstr. 15, 12489 Berlin, Germany

187. Texas Tech University, 2500 Broadway, Lubbock, Texas 79409-1035, USA

188. University of Zielona Góra, ul. Licealna 9, 65-417 Zielona Góra, Poland

189. Institute for Nuclear Research and Nuclear Energy, Bulgarian Academy of Sciences, 72 boul. Tsarigradsko chaussee, 1784 Sofia, Bulgaria

190. University of Białystok, Faculty of Physics, ul. K. Ciołkowskiego 1L, 15-254 Białystok, Poland

191. Faculty of Physics, National and Kapodestrian University of Athens, Panepistimiopolis, 15771 Ilissia, Athens, Greece

192. Universidad de Chile, Av. Libertador Bernardo O'Higgins 1058, Santiago, Chile

193. Hiroshima Astrophysical Science Center, Hiroshima University, Higashi-Hiroshima, Hiroshima 739-8526, Japan

194. Department of Applied Physics, University of Miyazaki, 1-1 Gakuen Kibana-dai Nishi, Miyazaki, 889-2192, Japan

195. School of Allied Health Sciences, Kitasato University, Sagamihara, Kanagawa 228-8555, Japan

196. Departamento de Astronomía, Universidad de Concepción, Barrio Universitario S/N, Concepción, Chile

197. Charles University, Institute of Particle \& Nuclear Physics, V Holešovičkách 2, 18000 Prague 8, Czech Republic

198. Astronomical Observatory of Ivan Franko National University of Lviv, 8 Kyryla i Mephodia Street, Lviv, 79005, Ukraine

199. Kobayashi-Maskawa Institute (KMI) for the Origin of Particles and the Universe, Nagoya University, Chikusa-ku, Nagoya 464-8602, Japan

200. Graduate School of Technology, Industrial and Social Sciences, Tokushima University, Tokushima 770-8506, Japan

201. Space Research Centre, Polish Academy of Sciences, ul. Bartycka 18A, 00-716 Warsaw, Poland

202. Instituto de Física - Universidade de São Paulo, Rua do Matão Travessa R Nr.187 CEP 05508-090 Cidade Universitária, São Paulo, Brazil

203. International Institute of Physics at the Federal University of Rio Grande do Norte, Campus Universitário, Lagoa Nova CEP 59078-970 Rio Grande do Norte, Brazil

204. University College Dublin, Belfield, Dublin 4, Ireland

205. Centre for Astro-Particle Physics (CAPP) and Department of Physics, University of Johannesburg, PO Box 524, Auckland Park 2006, South Africa

206. Departamento de Física, Facultad de Ciencias Básicas, Universidad Metropolitana de Ciencias de la Educación, Santiago, Chile

207. Núcleo de Formação de Professores - Universidade Federal de São Carlos, Rodovia Washington Luís, km 235 CEP 13565-905 - SP-310 São Carlos - São Paulo, Brazil

208. Physik-Institut, Universität Zürich, Winterthurerstrasse 190, 8057 Zürich, Switzerland

209. Department of Physical Sciences, Aoyama Gakuin University, Fuchinobe, Sagamihara, Kanagawa, 252-5258, Japan

210. University of the Free State, Nelson Mandela Avenue, Bloemfontein, 9300, South Africa

211. Faculty of Electronics and Information, Warsaw University of Technology, ul. Nowowiejska 15/19, 00-665 Warsaw, Poland

212. Rudjer Boskovic Institute, Bijenicka 54, 10000 Zagreb, Croatia

213. Department of Physics, Konan University, Kobe, Hyogo, 658-8501, Japan

214. Kumamoto University, 2-39-1 Kurokami, Kumamoto, 860-8555, Japan

215. University School for Advanced Studies IUSS Pavia, Palazzo del Broletto, Piazza della Vittoria 15, 27100 Pavia, Italy

216. Aalto University, Otakaari 1,00076 Aalto, Finland

217. Agenzia Spaziale Italiana (ASI), 00133 Roma, Italy

218. Observatoire de la Cote d'Azur, Boulevard de l'Observatoire CS34229, 06304 Nice Cedex 4, Franc 